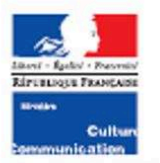

Secretarlat general

Service de la

coordination des

polltiques cultureles

et de ilinnovation

Departement

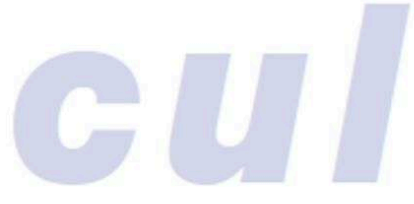

82, rue Saint-Honoré, 75033 Paris cedex 01

\title{
Les activités marchandes de spectacle vivant en 2010
}

Valérie Deroin

\author{
The market-oriented sectors \\ of performing arts in 2010
}

En 2010, les entreprises marchandes de spectacle vivant réalisent un chiffre d'affaires de 4,4 milliards d'euros et dégagent une valeur ajoutée de près de 1,5 milliard d'euros. En dépit d'une grande variété de situations individuelles, des caractéristiques communes ressortent : le tissu est dominé par de petites structures, très fortement implantées en Île-de-France, dont la taille modeste va de pair avec un fort dynamisme en termes de valeur produite : près de la moitié du chiffre d'affaires du spectacle vivant est réalisé par les entreprises de moins de 10 salariés. L'importance des coûts liés au travail et à la production des spectacles apparaît, ainsi que des investissements matériels notables et des activités centrées sur leur çeur de métier. Au final, les indicateurs de rentabilité ou de performance économique sont, dans le spectacle vivant marchand, en deçà de ceux de l'ensemble des services marchands. Le spectacle vivant marchand contribue à $11 \%$ du nombre d'unités des secteurs culturels marchands, $5 \%$ de leur valeur ajoutée et $3 \%$ de leur excédent brut d'exploitation.

Les unités marchandes du spectacle vivant ont été enquêtées pour la première fois en 2011 dans le cadre du dispositif de la statistique d'entreprise de l'Insee (élaboration des statistiques annuelles d'entreprises, Ésane ${ }^{1}$ ). La présente publication propose un traitement des nouvelles données portant sur les résultats structurels et comptables de 2010 , complétés par des questions spécifiques au spectacle vivant. Son objectif est de produire un premier panorama des caractéristiques économiques principales des trois secteurs d'activités du spectacle vivant, tels qu'ils sont définis par la nomenclature d'activités française NAF Rév.2, 2008 : les activités des arts du spectacle vivant (code NAF 90.01Z), celles de soutien au spectacle vivant (code NAF 90.02Z) et celles de gestion des salles de spectacles (code NAF 90.04Z).

Ces trois secteurs d'activités permettent de prendre en compte la variété des entreprises intervenant dans le spectacle vivant. Le champ intègre ainsi les producteurs de spectacles, les organisateurs avec ou sans la responsabilite d'employeur, les diffuseurs, les exploitants marchands de lieux culturels ou polyvalents mais également les entrepreneurs administratifs spécialisés tels les bureaux de production ou encore les entrepreneurs techniques et ceux ayant des activités de services spécifiques dédiées au spectacle vivant.

Les données présentées excluent les entreprises non marchandes du spectacle vivant, y compris celles employant un grand nombre de salariés. Ces entreprises non marchandes sont principalement des scènes subventionnées, exclues par leur statut juridique, et/ou des associations contribuant en moyenne plus faiblement à la valeur ajoutée marchande du secteur ${ }^{2}$.

Cette restriction aux entreprises marchandes est parfois délicate : en effet, la caractéristique du spectacle vivant est de présenter une hybridation forte entre le marchand et le

* Valérie Deroin est charóée d'analyses statistiques au preps.

1. Voir Ésane, Insee : http://wwwinsee.ff/fr/bases-de-donnees/default.asp?pase=presentation-stat-annuelle-entreprise htm

1. Ves Essociations du spectacle vivant feront l'objet d'une prochaine publication du priss en 2013, à partir des données de l'enquete $\propto$ Vie associative

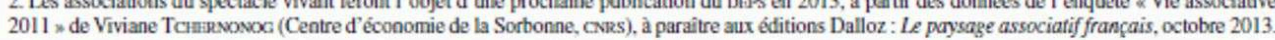




\section{Les activités marchandes de spectacle vivant en 2010}

The market-oriented sectors of performing arts in 2010

\section{Valérie Deroin}

Éditeur : Département des études, de la prospective et des statistiques

Lieu d'édition : Paris

Année d'édition : 2013

Date de mise en ligne : 21 septembre 2015

Collection : Culture chiffres

ISBN électronique : 9782111398627

\section{Sobooks}

http://books.openedition.org

\section{Édition imprimée}

Date de publication : 1 avril 2013

Nombre de pages : 16

\section{Référence électronique}

DEROIN, Valérie. Les activités marchandes de spectacle vivant en 2010. Nouvelle édition [en ligne]. Paris :

Département des études, de la prospective et des statistiques, 2013 (généré le 25 avril 2021).

Disponible sur Internet : <http://books.openedition.org/deps/487>. ISBN : 9782111398627. 


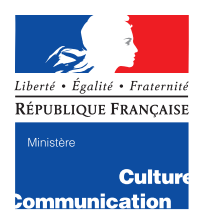

Secrétariat général

Service de la

coordination des

politiques culturelles

et de l'innovation

Département

des études,

de la prospective

et des statistiques
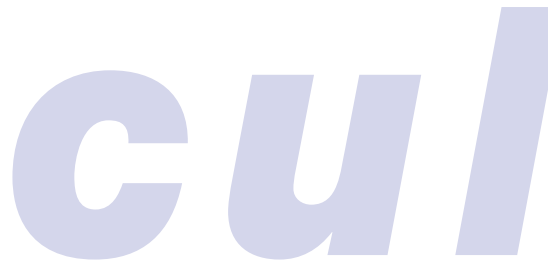

182, rue Saint-Honoré, 75033 Paris cedex 01 용 0140157917 - 圆 0140157999

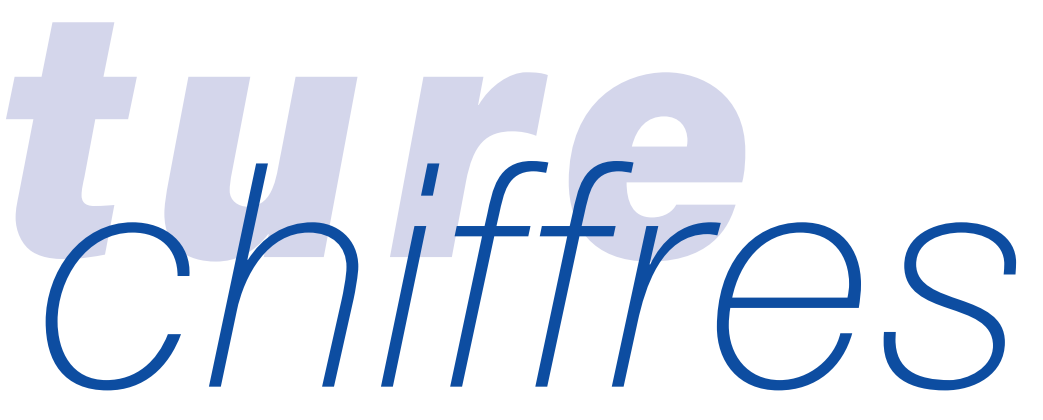

ÉCONOMIE DE LA CULTURE ET DE LA COMMUNICATION

Téléchargeable sur le site http://www.culturecommunication.gouv.fr/deps

2013-1

\title{
Les activités marchandes de spectacle vivant en 2010
}

\author{
Valérie Deroin
}

\section{The market-oriented sectors of performing arts in 2010}

En 2010, les entreprises marchandes de spectacle vivant réalisent un chiffre d'affaires de 4,4 milliards d'euros et dégagent une valeur ajoutée de près de 1,5 milliard d'euros. En dépit d'une grande variété de situations individuelles, des caractéristiques communes ressortent : le tissu est dominé par de petites structures, très fortement implantées en Île-de-France, dont la taille modeste va de pair avec un fort dynamisme en termes de valeur produite : près de la moitié du chiffre d'affaires du spectacle vivant est réalisé par les entreprises de moins de $\mathbf{1 0}$ salariés. L'importance des coûts liés au travail et à la production des spectacles apparaît, ainsi que des investissements matériels notables et des activités centrées sur leur cœur de métier. Au final, les indicateurs de rentabilité ou de performance économique sont, dans le spectacle vivant marchand, en deçà de ceux de l'ensemble des services marchands. Le spectacle vivant marchand contribue à $11 \%$ du nombre d'unités des secteurs culturels marchands, $5 \%$ de leur valeur ajoutée et $3 \%$ de leur excédent brut d'exploitation.

Les unités marchandes du spectacle vivant ont été enquêtées pour la première fois en 2011 dans le cadre du dispositif de la statistique d'entreprise de l'Insee (élaboration des statistiques annuelles d'entreprises, Ésane ${ }^{1}$ ). La présente publication propose un traitement des nouvelles données portant sur les résultats structurels et comptables de 2010, complétés par des questions spécifiques au spectacle vivant. Son objectif est de produire un premier panorama des caractéristiques économiques principales des trois secteurs d'activités du spectacle vivant, tels qu'ils sont définis par la nomenclature d'activités française NAF Rév.2, 2008 : les activités des arts du spectacle vivant (code NAF 90.01Z), celles de soutien au spectacle vivant (code NAF 90.02Z) et celles de gestion des salles de spectacles (code NAF 90.04Z).

Ces trois secteurs d'activités permettent de prendre en compte la variété des entreprises intervenant dans le spectacle vivant. Le champ intègre ainsi les producteurs de spectacles, les organisateurs avec ou sans la responsabilité d'employeur, les diffuseurs, les exploitants marchands de lieux culturels ou polyvalents mais également les entrepreneurs administratifs spécialisés tels les bureaux de production ou encore les entrepreneurs techniques et ceux ayant des activités de services spécifiques dédiées au spectacle vivant.

Les données présentées excluent les entreprises non marchandes du spectacle vivant, y compris celles employant un grand nombre de salariés. Ces entreprises non marchandes sont principalement des scènes subventionnées, exclues par leur statut juridique, et/ou des associations contribuant en moyenne plus faiblement à la valeur ajoutée marchande du secteur ${ }^{2}$.

Cette restriction aux entreprises marchandes est parfois délicate : en effet, la caractéristique du spectacle vivant est de présenter une hybridation forte entre le marchand et le

\footnotetext{
* Valérie Deroin est chargée d'analyses statistiques au DEPS.

1. Voir Ésane, Insee : http://www.insee.fr/fr/bases-de-donnees/default.asp?page=presentation-stat-annuelle-entreprise.htm

2. Les associations du spectacle vivant feront l'objet d'une prochaine publication du DEPS en 2013, à partir des données de l'enquête « Vie associative

2011 » de Viviane TCHERNONOG (Centre d'économie de la Sorbonne, CNRS), à paraître aux éditions Dalloz : Le paysage associatiffrançais, octobre 2013.
} 
non-marchand ; par ailleurs, une entreprise majoritairement non marchande produit, même très faiblement, de la valeur ajoutée marchande. Au final, les données ici présentées couvrent $80 \%$ du chiffre d'affaires du spectacle vivant ${ }^{3}$. La restriction au champ marchand permet de comparer les résultats économiques du spectacle vivant à celles des entreprises marchandes culturelles ou, plus globalement, à d'autres secteurs de l'économie marchande française et, à terme, de suivre annuellement l'évolution du poids économique du spectacle vivant.

\section{Le champ des entreprises marchandes du spectacle vivant}

\section{Quel est le champ de l'analyse économique du spectacle vivant?}

L'ordonnance n 45-2339 du 13 octobre 1945, modifiée par la loi du 18 mars 1999 définit le spectacle vivant professionnel en tant que "spectacles vivants produits ou diffusés par des personnes qui, en vue de la représentation en public d'une œuvre de l'esprit, s'assurent la présence physique d'au moins un artiste du spectacle percevant une rémunération ". Le spectacle vivant professionnel résulte donc de la présence physique d'un artiste du spectacle rémunéré, de l'interprétation d'une œuvre de l'esprit et d'un public composé de la présence physique de spectateurs.

Quant aux activités d'entrepreneurs de spectacles vivants, elles sont délimitées à travers trois métiers décrits par la circulaire du 13 juillet 2000 : le métier d'exploitant de salles (licence 1), celui de producteur de spectacles (licence 2) et celui de diffuseur (licence 3). L'obtention des licences est cumulable.

L'analyse de l'activité économique ${ }^{1}$ du spectacle vivant suppose de disposer de codes de référence pour classifier ces activités et pouvoir les suivre dans le temps et les comparer à d'autres activités: c'est le rôle des activités statistiques qui s'organisent à partir de la nomenclature d'activités française de 2008 (NAF Rév.2, 2008), norme servant à l'analyse et à la diffusion des résultats des secteurs d'activités de l'économie française.

Les trois codes NAF relatifs au spectacle vivant ne reposent pas uniquement sur les concepts d'entrepreneurs de spectacles vivants et ne différencient pas strictement les trois métiers tels qu'ils le sont dans les licences d'entrepreneurs. Ils couvrent toute la chaîne en incluant des activités dites "de soutien " au spectacle vivant: entreprises de prestations techniques (exemples: scénographes, entreprises de sonorisation), des bureaux de production (exemples: conseils en diffusion, recherche de partenaires, services administratifs), des sociétés de perception et de répartition des droits.

C'est par l'attribution d'un code d'activité principale exercée (APE) que les entreprises sont classées pour faire partie d'un secteur de la NAF : une entreprise ne peut avoir qu'une seule APE, attribuée et calculée à partir de la répartition de son chiffre d'affaires. Par exemple, une entreprise d'exploitation de salles de spectacles a différentes activités, dont celle de production de spectacles, de location de salles ou encore de restauration et débit de boissons : son APE sera attribuée en fonction de l'activité la plus contributrice au total de son chiffre d'affaires. Ce code APE peut être mis à jour grâce à l'enquête sectorielle annuelle et les questions sur la ventilation du chiffre d'affaires.

Le secteur d'activités regroupe les entreprises qui ont le même code d'activité principale exercée.

1. L'activité économique d'une entreprise est le processus qui conduit à la fabrication d'un produit ou à la mise à disposition d'un service. Les activités économiques en France sont organisées à travers la nomenclature d'activités française (NAF), la NAF Rév. 2, 2008 en vigueur depuis le $1^{\text {er }}$ janvier 2008.

Les « activités » ne doivent pas être confondues avec les «professions » : une profession correspond à un métier.

\section{Quel est le contenu des secteurs d'activités du spectacle vivant?}

Trois secteurs d'activités de la nomenclature d'activités française NAF Rév.2, 2008 permettent d'identifier les entreprises du spectacle vivant. II s'agit des codes :

\section{$90.01 Z$ - Arts du spectacle vivant}

Ce secteur d'activités comprend:

- la production de spectacles, de productions théâtrales, de concerts, de spectacles d'opéra, de spectacles de danse et d'autres productions analogues (activités de groupes, de cirques ou de compagnies, d'orchestres ou d'autres formations ; activités exercées par des artistes indépendants tels que des acteurs, danseurs, musiciens, conteurs);

- l'organisation de tournées et la diffusion de spectacles lorsqu'elles comprennent la responsabilité artistique du spectacle ;

- l'activité des conférenciers.

$90.02 Z$ - Activités de soutien au spectacle vivant

- les activités de soutien au spectacle vivant assurant des prestations de services techniques pour le son, l'éclairage, le décor, le montage de structures, la projection d'images ou de vidéos, les costumes, etc. ;

- les activités de production, de promotion et d'organisation de spectacles ne comprenant pas la responsabilité artistique du spectacle.

\section{$90.04 Z$ - Gestion de salles de spectacles}

- l'exploitation de centres polyvalents et d'installations similaires à prédominance culturelle (par exemple, espaces de location d'événementiel, salles polyvalentes) ou l'exploitation de salles de spectacles aménagés pour des représentations publiques: salles de concert, de théâtre, de danse, de music hall, cirques, etc. (par exemple, salles de spectacles privées, sous statut particulier comme les Zénith, cafés-concerts, théâtres privés).

\section{D’où viennent les données ?}

Un dispositif multisources: l'enquête sectorielle annuelle (ESA) et le dispositif des statistiques d'entreprises de l'Insee

Le dispositif d'élaboration des statistiques annuelles d'entreprises (Ésane) de l'Insee permet de publier des données sur les sociétés et entreprises individuelles non financières de l'économie marchande en France : ce dispositif est construit sur deux sources, d'un côté les sources administratives (fichiers fiscaux des déclarations annuelles sur les bénéfices et fichiers sociaux des déclarations annuelles de données sociales - DADS) et de l'autre, les résultats d'enquête sur un échantillon d'entreprises, l'enquête sectorielle annuelle (ESA).

Ces sources ne peuvent pas être utilisées seules car elles se complètent en termes de champ couvert et en termes de variables.

Ainsi, l'enquête ESA permet de détailler les branches d'activités en ventilant le chiffre d'affaires annuel ou encore le type de clientèle et les dépenses; l'enquête permet aussi la mise à jour de l'activité 
principale exercée (APE). En effet, l'enquête ESA identifie les différentes activités exercées par les entreprises marchandes via la ventilation de leur chiffre d'affaires en branches. Cela permet de classer correctement les entreprises par secteur à partir de leur activité principale exercée (APE) et est essentiel à la production de statistiques sectorielles de qualité. L'enquête ESA permet alors de corriger des codes d'activités périmés : une unité du spectacle vivant qui était classée dans un secteur d'activités attribué lors de sa création, par exemple un commerce assimilé de restauration, pourrait avoir réorienté son activité vers la production de spectacles dont elle tirerait l'essentiel de ses revenus.

Organisée autour du tronc commun des questionnaires ESA, l'extension aux activités du spectacle vivant enrichit le questionnaire de cadres particuliers à ces secteurs: ventilation détaillée de leur chiffre d'affaires, emploi, dépenses particulières et type de clientèle.

Par ailleurs, la mise en cohérence des résultats de cette enquête avec des sources administratives sur la comptabilité et l'emploi permet d'alléger le questionnaire et de disposer de données sur toute la population marchande.

À travers ce dispositif Ésane, la synthèse des données individuelles fiscales et sociales et des données de l'enquête autorise la production de résultats agrégés dits "composites" pour l'ensemble des unités marchandes de chaque secteur d'activités.

Pour les secteurs du spectacle vivant, il s'agit de disposer de données statistiques globales et centralisées qui permettront dorénavant de produire annuellement des indicateurs économiques et financiers comparables à ceux d'autres secteurs.

C'est pourquoi, pour la première fois en 2010, les trois secteurs d'activités du spectacle vivant ont été introduits dans l'enquête sectorielle annuelle (ESA) conduite par l'Insee : cette intégration du spectacle vivant doit permettre à l'avenir de disposer de séries pour suivre l'évolution des secteurs marchands du spectacle vivant et les situer dans l'ensemble de l'économie française marchande.

\section{Qu'est-ce que le champ marchand?}

Une unité est considérée comme «marchande » quand elle tire l'essentiel de ses ressources de la vente de biens ou de services. À l'inverse, une unité «non marchande » assure son financement principalement par des ressources autres comme les subventions publiques ou les contributions privées (mécénat, dons, contributions volontaires de bénévoles, etc.).

En théorie, la règle est de dire qu'une unité rend des services marchands lorsqu'elle les vend à des prix économiquement significatifs et lorsque ses coûts de production sont couverts à plus de $50 \%$ par ses ventes.

En pratique, ce sont trois critères qui gèrent l'inclusion d'une structure dans le champ marchand : premièrement le code d'activité NAF, deuxièmement le statut juridique et troisièmement le fait pour une association de répondre à la règle comptable et d'être employeuse.

Ainsi, certains codes de catégories juridiques sont exclus du champ Ésane: les associés-gérants, les administrations et personnes morales de droit public ou encore les associations non employeuses sans activité commerciale (considérées alors comme non exploitantes).

Le champ marchand Insee repose donc sur l'existence de documents comptables (liasses fiscales, assujettissement ou déclaration aux impôts commerciaux) et la déclaration d'un effectif salarié au 31 décembre de l'année.

Du fait de ces critères, certaines structures du spectacle vivant employant un nombre important de salariés n'appartiennent donc pas au champ : par exemple les établissements publics de l'Opéra de Paris ou du Théâtre de Chaillot, des structures largement subventionnées ou des structures associatives sans emploi salarié déclaré.
Pour simplifier, il faut retenir que le champ Ésane décrit les secteurs marchands du spectacle vivant, hors associations non employeuses non marchandes. Bien que les résultats en nombre d'unités et d'emplois soient inférieurs à ceux couvrant le champ complet « marchand/non marchand ", ces résultats retracent bien le poids économique des secteurs puisque les unités marchandes sont les plus contributrices à la valeur ajoutée : en 2010, elles participent à environ $80 \%$ du chiffre d'affaires (source : référentiel SIRENE®).

\section{Comment est constitué l'échantillon « spectacle vivant » de l'ESA 2010 ?}

La base de sondage représente le socle des unités marchandes appartenant au champ de l'enquête: sur cette base, il convient chaque année de sélectionner les unités de l'échantillon qui recevront le questionnaire.

La mise en place d'un échantillon représentatif de l'ensemble de la population étudiée repose sur le choix des variables d'études (répartition du chiffre d'affaires dans notre cas) et le niveau de détail de diffusion souhaité (activité principale, niveau sous-classe de la nomenclature). L'échantillonnage est réalisé selon les principes appliqués à l'ESA avec une stratification qui consiste à croiser le code d'activité principale exercée (APE) des unités légales et leur tranche d'effectif (TEFF). Lors du tirage, les taux de sondage obtenus sont appliqués dans chaque région: pour un croisement APE $\times$ TEFF donné, chaque région sera représentée proportionnellement à son importance en nombre d'entreprises. Toutes les tailles d'entreprise sont dans le champ de l'enquête ${ }^{2}$. Pour alléger la charge statistique auprès des petites entreprises, la stratégie de l'enquête ESA est l'alternance: ne sont interrogées exhaustivement chaque année que les seules entreprises dont l'importance individuelle dans le secteur oblige à les inclure dans l'enquête.

Cette importance individuelle est définie à partir de seuils imposés qui permettent, de plus, d'améliorer l'échantillon dans chaque strate et, pour chaque secteur d'activité, d'obtenir la meilleure précision d'estimation du chiffre d'affaires total. Des seuils de chiffre d'affaires et d'effectifs salariés ont été calculés pour faire entrer une entreprise dans la partie exhaustive: les unités ayant un chiffre d'affaires important et un effectif faible sont ainsi mises à part, et les unités restantes ont alors des chiffres d'affaires plus homogènes au sein de chaque strate.

Pour l'ESA spectacle vivant, les seuils définis en 2010 sont les suivants:

- chiffre d'affaires minimum : $1000 \mathrm{k} €$;

- effectif salarié minimum: 30.

Ainsi, toute unité qui possède un chiffre d'affaires supérieur à 1 million d'euros ou un effectif salarié supérieur à 30 salariés ETP est sélectionnée dans la partie exhaustive de l'échantillon.

Quant aux unités en deçà de ces seuils d'exhaustivité, elles ne sont enquêtées par sondage qu'une année sur deux. La part exhaustive des trois secteurs du spectacle vivant occupe $50 \%$ de l'échantillon en 2010, taux moyen également pratiqué sur l'ensemble de l'ESA et supérieur au taux moyen des entreprises de services aux particuliers $(25 \%)$.

En 2010, le taux de sondage pour 1500 unités légales ${ }^{3}$ enquêtées dans les trois secteurs du spectacle vivant est de $12,1 \%$.

2. Tranches d'effectif salarié : $00: 0$ ou non-renseigné ; $01: 1$ à 5 salariés ; 03 : 6 à 9 salariés; $11: 10$ à 19 salariés; $12: 20$ à 29 salariés; $13: 30$ à 49 salariés; $21: 50$ à 99 salariés; $22: 100$ à 199 salariés; $23: 200$ salariés ou plus. 3. Parmi lesquelles, dans l'échantillon 2010, 16 associations (soit association déclarée, soit association de droit local) et 220 entreprises individuelles (avec des statuts juridiques d'artisans, de commerçants, de professions libérales, ou "autres personnes physiques "). 


\section{Panorama Comptable général DU SPECTACLE VIVANT}

\section{Le spectacle vivant représente $11 \%$ des unités marchandes des secteurs culturels ${ }^{4}$, $6 \%$ de leur chiffre d'affaires et $5 \%$ de leur valeur ajoutée}

Avec environ 18000 unités marchandes et 20000 salariés en équivalent temps plein (ETP), les trois secteurs d'activités marchandes du spectacle vivant réalisent, en 2010, un chiffre d'affaires de 4,4 milliards d'euros et dégagent une valeur ajoutée de près de 1,5 milliard d'euros (tableau 1).

Plus d'une entreprise culturelle marchande sur dix est rattachée au spectacle vivant : cette part relativement élevée est due à la forte présence, dans le spectacle vivant, de petites structures employant peu de salariés par comparaison à d'autres activités culturelles (audiovisuel, édition par exemple). C'est notamment le cas dans les arts du spectacle vivant, qui comptent plus de 11000 unités marchandes en 2010 , contribuant ainsi pour plus de $60 \%$ au nombre d'unités du spectacle vivant, mais seulement pour $40 \%$ de son chiffre d'affaires.

Les structures des arts du spectacle sont formées de producteurs de spectacles (exemples : groupes, cirques ou compagnies, orchestres ou autres formations, artistes indépendants tels que acteurs, danseurs, musiciens, conteurs), d'organisateurs de spectacles et d'entrepreneurs de tournées avec responsabilité artistique (notamment employeurs du plateau artistique).

Bien que deux fois moins nombreuses, les structures de soutien au spectacle contribuent dans les mêmes proportions que les entreprises des arts du spectacle vivant au chiffre d'affaires de l'ensemble du spectacle vivant (environ pour $40 \%$ ) et un peu plus de la valeur ajoutée (environ $48 \%$ en 2010 contre $40 \%$ pour les arts du spectacle vivant).

À l'opposé, les structures marchandes de gestion de salles de spectacles sont les moins nombreuses : toutefois, si elles ne représentent qu'environ $3 \%$ du nombre d'unités légales marchandes du spectacle vivant, elles contribuent pour $22 \%$ au chiffre d'affaires et pour $13 \%$ à la valeur ajoutée en 2010 (graphique 1).

Afin de calculer des ratios d'emploi comparables, l'emploi mesuré en équivalent temps plein (ETP) est l'indicateur habituellement utilisé : l'emploi salarié ETP cumule les heures de tous les postes de travail des salariés y compris les postes à temps partiel (contrats à durée indéterminée, contrats à durée déterminée, contrats à durée déterminée d'usage), en les rapportant à la moyenne annuelle des heures travaillées dans les emplois à plein temps. La norme qui s'applique est celle de la durée légale du travail salarié de l'ensemble de l'économie marchande: cette convention conduit à faire abstraction des différentes intensités de travail, comme cela peut exister dans les secteurs du spectacle vivant marchand. L'effectif salarié ETP doit donc être vu comme un indicateur intermédiaire de calcul et de macroéconomie 5 .

Tableau 1 - Chiffres clés du spectacle vivant marchand en 2010

\begin{tabular}{|c|c|c|c|c|c|c|c|}
\hline $\begin{array}{l}\text { Secteur } \\
\text { d'activité NAF } \\
2008\end{array}$ & Libellé de l'activité & $\begin{array}{l}\text { Nombre } \\
\text { d'unités } \\
\text { légales }\end{array}$ & $\begin{array}{l}\text { Effectifs } \\
\text { salariés } \\
\text { en équivalent } \\
\text { temps plein }\end{array}$ & $\begin{array}{l}\text { Effectifs } \\
\text { salariés } \\
\text { au } 31 \\
\text { décembre }\end{array}$ & $\begin{array}{c}\text { Chiffre } \\
\text { d'affaires } \\
\text { hors taxes } \\
\text { (en millions d'euros) }\end{array}$ & $\begin{array}{c}\text { Chiffre } \\
\text { d'affaires } \\
\text { à l'exportation } \\
\text { (en millions d'euros) }\end{array}$ & $\begin{array}{c}\text { Valeur ajoutée } \\
\text { y compris } \\
\text { autres produits } \\
\text { et autres } \\
\text { charges } \\
\text { (en millions d'euros) }\end{array}$ \\
\hline $90.01 Z$ & Arts du spectacle vivant & 11097 & 8090 & 9380 & 1721 & 118 & 574 \\
\hline $90.02 Z$ & Activités de soutien au spectacle vivant & 5922 & 8522 & 10909 & 1759 & 134 & 694 \\
\hline \multirow[t]{4}{*}{$90.04 Z$} & Gestion de salles de spectacles & 514 & 3739 & 4279 & 959 & 19 & 183 \\
\hline & Total spectacle vivant marchand & 17533 & 20351 & 24568 & 4439 & 271 & 1451 \\
\hline & $\begin{array}{l}\text { Total secteurs culturels marchands* } \\
\text { soit en \% des secteurs }\end{array}$ & 156579 & 280984 & 341291 & 70814 & 5580 & 28656 \\
\hline & culturels marchands & $11 \%$ & $7 \%$ & $7 \%$ & $6 \%$ & $5 \%$ & $5 \%$ \\
\hline
\end{tabular}

* Les secteurs culturels incluent l'édition de livres, de journaux, de revues et périodiques, de jeux électroniques; la production de films et programmes pour la télévision de films institutionnels et publicitaires, de films pour le cinéma; la postproduction; la distribution de films ; l'édition et la distribution vidéo ; la projection de films ; l'édition musicale; l'édition et la diffusion de programmes radio; l'édition de chaînes généralistes et thématiques; les agences de presse et de publicité; les activités d'architecture, de design, de photographie ; la création artistique ; les arts du spectacle vivant, les activités de soutien au spectacle vivant, les salles de spectacles ; l'enseignement artistique et de loisirs ; les bibliothèques, archives et sites et monuments historiques (codes NAF suivants : $58.11 Z, 58.13 Z, 58.14 Z, 58.21 Z, 59.11 \mathrm{~A}$, 59.11B, 59.11C, 59.12Z, 59.13A, 59.13B, 59.14Z, 59.20Z, 60.10Z, 60.20A, 60.20B, 63.91Z, 71.11Z, 73.11Z, 74.10Z, 74.20Z, 85.52Z, 90.01Z, 90.02Z, 90.03A, 90.03B, 90.04Z, 91.01Z, 91.02Z, 91.03Z).

Champ : France, ensemble des unités légales des secteurs marchands, y compris auto-entrepreneurs.

4. Les secteurs culturels correspondent aux activités du patrimoine, du livre et de la presse, des arts visuels, de l'architecture, du spectacle vivant, de l'audiovisuel et du multimédia, de la publicité et de l'enseignement artistique et de loisirs, tels que définis dans la publication Repères économiques des secteurs culturels marchands en 2010, Paris, Ministère de la Culture et de la Communication, DEPS, coll. « Culture chiffres », $2012-3$, octobre 2012. 5. Des données détaillées sur l'emploi salarié sont publiées dans les collections du DEPS par Marie GouYON et Frédérique PATUREAU, voir par exemple le Salariat dans le secteur culturel en 2009 : flexibilité et pluriactivité, Paris, Ministère de la Culture et de la Communication, DEPS, coll. « Culture chiffres », 2012-2, septembre 2012. 
Graphique 1 - Poids des trois secteurs du spectacle vivant en termes d'effectifs salariés ETP, de chiffre d'affaires et de valeur ajoutée en 2010

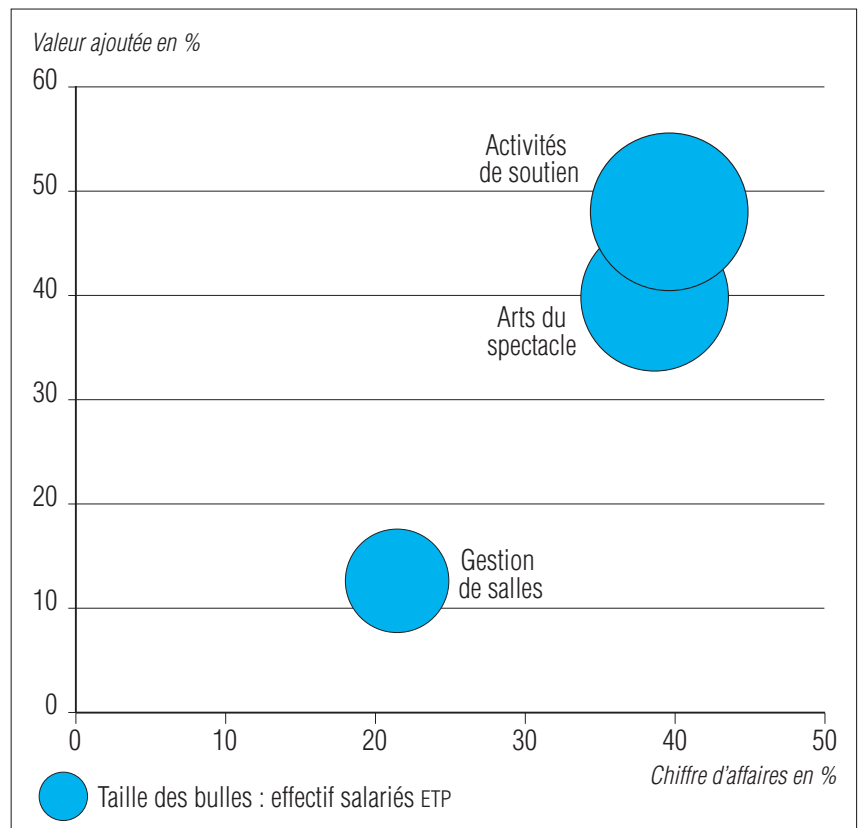

Champ: France, ensemble des unités légales des secteurs marchands, y compris auto-entrepreneurs.

Note de lecture : au sein du spectacle vivant, l'activité marchande de gestion de salles contribue pour $22 \%$ à son chiffre d'affaires et pour $15 \%$ à la valeur ajoutée de l'ensemble du champ économique.

Source : Insee, Ésane 2010/Ministère de la Culture et de la Communication, DEPS, 2013

Dans l'ensemble du spectacle vivant, la part du chiffre d'affaires réalisé à l'exportation (6\% en 2010) est légèrement inférieure à celle observée dans l'ensemble du secteur culturel marchand $(8 \%)$ ou, plus globalement, à celle relative à l'ensemble des services marchands $(9 \%)$. Ce résultat masque des disparités : dans les arts du spectacle vivant et des activités de soutien, il correspond aux taux moyens rencontrés dans les autres secteurs culturels, soit environ $8 \%$, tandis qu'il est moindre pour les salles de spectacles, et similaire à celui des autres activités de services en général (spectacles, patrimoine, activités récréatives, services aux particuliers), soit $2 \%$ en 2010.

La valeur ajoutée dégagée, soit la différence entre la valeur finale de la production (exprimée par le chiffre d'affaires) et la valeur des biens consommés par le processus de production (consommations intermédiaires de biens et services, autres charges), s'élève pour l'ensemble du spectacle vivant à un peu plus de 1,4 milliard d'euros en 2010. Elle est équivalente à celle produite par le secteur de production de films pour le cinéma (1,4 milliard d'euros en 2010) ou encore représente le double de celle des activités de design (environ 700 millions d'euros en 2010) et presque la moitié de la valeur ajoutée engendrée par les éditeurs de chaînes généralistes (3,1 milliards d'euros en 2010).

\section{Un taux de marge plutôt faible, en particulier dans la gestion de salles de spectacles ou les activités de soutien}

La richesse produite par les entreprises du spectacle vivant se répartit entre différents partenaires de l'entreprise, parmi lesquels l'État et les collectivités territoriales (par le paiement des impôts, taxes et versements assimilés) et les salariés (par le paiement des salaires et charges sociales). Le reste permet à l'entreprise de générer des ressources à la fois pour le paiement de ses autres charges et pour son autofinancement. C'est ce que mesure l'excédent brut d'exploitation (EBE), augmenté des subventions d'exploitation qui permettent à l'entreprise de compenser l'insuffisance de certains produits d'exploitation ou de faire face à certaines charges d'exploitation (tableau 2). Par exemple, en comparant la valeur ajoutée à l'excédent brut d'exploitation, on constate que la richesse produite par les salles de spectacles diminue plus fortement que celle des deux autres secteurs : en 2010, les frais de personnel des salles de spectacles sont d'un montant supérieur à la valeur ajoutée et grèvent donc de façon importante la richesse produite. L'excédent brut d'exploitation des salles de spectacles affiche cependant un résultat positif en 2010 grâce à la compensation apportée par les subventions d'exploitation.

Tableau 2 - Taux de marge du spectacle vivant en 2010

\begin{tabular}{|c|c|c|c|c|c|c|c|}
\hline $\begin{array}{l}\text { Secteur } \\
\text { d'activité NAF } \\
2008\end{array}$ & Libellé de l'activité & $\begin{array}{l}\text { Valeur ajoutée } \\
\text { (VA) } \\
\text { (en millions d'euros) }\end{array}$ & $\begin{array}{c}+ \\
\text { Subventions } \\
\text { d'exploitation } \\
\text { (en millions d'euros) }\end{array}$ & $\begin{array}{c}- \\
\text { Impôts, } \\
\text { taxes et } \\
\text { versements } \\
\text { assimilés } \\
\text { (en millions d'euros) }\end{array}$ & $\begin{array}{c}\text { - } \\
\text { Frais } \\
\text { de personnel } \\
\text { (en millions d'euros) }\end{array}$ & $\begin{array}{c}= \\
\text { Excédent } \\
\text { brut } \\
\text { d'exploitation } \\
\text { (EBE) } \\
\text { (en millions d'euros) }\end{array}$ & $\begin{array}{c}\text { Taux } \\
\text { de marge } \\
\text { (en } \%)\end{array}$ \\
\hline $90.01 Z$ & Arts du spectacle vivant & 574 & 152 & 38 & 545 & 144 & 25 \\
\hline $90.02 Z$ & Activités de soutien au spectacle vivar & ant 694 & 13 & 26 & 607 & 73 & 11 \\
\hline \multirow[t]{4}{*}{$90.04 Z$} & Gestion de salles de spectacles & 183 & 86 & 24 & 230 & 17 & 9 \\
\hline & Total spectacle vivant marchand & 1451 & 251 & 88 & 1381 & 234 & 16 \\
\hline & $\begin{array}{l}\text { Total secteurs culturels marchands } \\
\text { soit en \% des secteurs }\end{array}$ & 28656 & 1137 & 1541 & 19883 & 8369 & 29 \\
\hline & culturels marchands & $5 \%$ & $22 \%$ & $5 \%$ & $7 \%$ & $3 \%$ & - \\
\hline
\end{tabular}

Champ : France, ensemble des unités légales des secteurs marchands, y compris auto-entrepreneurs. 
L'EBE est donc le résultat de l'activité commerciale courante de l'entreprise. Rapporté à la valeur ajoutée, il fournit un indicateur comptable de rentabilité : le taux de marge. Le taux de marge moyen de l'ensemble du spectacle vivant est égal à $16 \%$, ce qui correspond à un taux nettement inférieur à celui des secteurs culturels marchands $(29 \%)$ ou de l'ensemble des secteurs marchands (27\%). Cependant, cette moyenne cache des différences : dans les arts du spectacle, le taux de marge est comparable à la moyenne des secteurs marchands $(25 \%)$ alors qu'il est sensiblement plus faible dans les activités de soutien et les salles de spectacles. Ces deux secteurs affichent des taux de marge analogues (respectivement $11 \%$ et $9 \%$ ) que l'on peut comparer à ceux des activités d'édition et de diffusion des radios $(8 \%$ en 2010) ou des agences de publicité (12\% en 2010).

Pour les trois secteurs du spectacle vivant, le résultat net comptable, soit la différence entre les produits et les charges de l'exercice après prise en compte de la dépréciation du capital, est positif en 2010 (tableau 3). Les trois secteurs du spectacle vivant réalisent cependant des bénéfices plus ou moins importants : ainsi les arts du spectacle font apparaître un bénéfice de 78 millions d'euros en 2010 alors que les salles de spectacles dégagent 27 millions d'euros et les activités de soutien 24 millions. Par comparaison, la production de films pour le cinéma réalise un bénéfice d'environ 100 millions d'euros en 2010.

Au contraire du résultat net comptable, la capacité d'autofinancement (CAF) ne prend pas en compte les flux se rapportant aux opérations d'appréciation ou de dépréciation du capital : elle représente la ressource dégagée au cours de l'exercice, qui reste à la disposition de l'entreprise. Cette ressource peut servir à développer l'entreprise en finançant les outils de production existants (soit par de nouveaux investissements, soit en contribuant à rembourser des emprunts) ou peut être distribuée (versements aux actionnaires, par exemple). La capacité d'autofinancement des arts du spectacle est de 125 millions d'euros en 2010, celles des activités de soutien de 62 millions d'euros et celles des salles de 4 millions d'euros (tableau 3) : le total de capacité d'autofinancement du spectacle vivant représente $5 \%$ du total de la CAF des secteurs culturels marchands.

En termes comptables, trois grands postes décrivent les types d'investissements : investissements corporels, investissements incorporels et investissements financiers. Les investissements corporels recouvrent tous les équipements (mobiliers, matériel divers, installations techniques) et infrastructures (bâtiments, terrains...) alors que les investissements incorporels concernent, par exemple, des frais de recherche et développement, des dépôts de brevets, licences ou marques et que les investissements financiers correspondent aux titres financiers ou demandes de prêts.

Dans les trois secteurs, les logiques d'investissements donnent, en 2010, la priorité aux investissements d'équipements et d'installations nécessaires à la mise en œuvre des activités finales de production et de représentation des spectacles : les investissements corporels représentent $60 \%$ à $70 \%$ du total des investissements alors que les investissements incorporels et financiers en prennent de $5 \%$ à $20 \%$. Par comparaison, dans l'édition de livres et de journaux, ce sont les investissements financiers qui concentrent l'essentiel des investissements de ces secteurs (entre $60 \%$ et $80 \%$ d'investissements financiers contre seulement $10 \%$ à $20 \%$ d'investissements corporels). Dans les activités de soutien, ce sont en particulier les investissements de matériels techniques qui sont importants ; il s'agit plutôt des installations générales et d'aménagements dans les salles de spectacles et des constructions et immobilisations corporelles en cours dans les arts du spectacle.

\section{Des charges d'activités essentiellement tournées vers les dépenses externes et d'exploitation}

Les dépenses engagées auprès de tiers pour la production de l'activité, la logistique et les services externes sont plus élevées que dans les autres activités culturelles marchandes.

Hors impôts, taxes et charges de personnel, les trois premiers comptes de charges des activités de spectacle vivant sont dus aux charges externes, aux achats de matières premières et aux charges de location (tableau 4). Les autres achats et charges externes concernent autant les dépenses liées aux achats de spectacles (contrats) que les dépenses de services extérieurs (services techniques, frais de communication, voyages, hébergement, etc.). Ces charges représentent le coût le plus lourd pour les activités des arts du spectacle en 2010 , soit $51 \%$ du total des charges hors impôts, taxes et charges de personnel : à l'instar de deux autres secteurs culturels, les éditions de revues et périodiques $(56 \%)$ et la production de films institutionnels et

Tableau 3 - Résultat net comptable et investissements du spectacle vivant en 2010

\begin{tabular}{|c|c|c|c|c|c|c|}
\hline $\begin{array}{l}\text { Secteur } \\
\text { d'activité NA } \\
2008\end{array}$ & Libellé de l'activité & $\begin{array}{l}\text { Capacité } \\
\text { d'autofinancement }\end{array}$ & $\begin{array}{l}\text { Résultat net } \\
\text { comptable }\end{array}$ & $\begin{array}{l}\text { Investissements } \\
\text { financiers }\end{array}$ & $\begin{array}{l}\text { Investissements } \\
\text { incorporels }\end{array}$ & $\begin{array}{l}\text { Investissements } \\
\text { corporels }\end{array}$ \\
\hline $90.01 Z$ & Arts du spectacle vivant & 125 & 78 & 16 & 16 & 49 \\
\hline $90.02 Z$ & Activités de soutien au spectacle vivant & 62 & 24 & 13 & 12 & 80 \\
\hline \multirow[t]{4}{*}{$90.04 Z$} & Gestion de salles de spectacles & 4 & 27 & 1 & 5 & 15 \\
\hline & Total spectacle vivant marchand & 191 & 129 & 29 & 33 & 143 \\
\hline & Total secteurs culturels marchands & 7944 & 2721 & 2818 & 6521 & 1380 \\
\hline & soit en $\%$ des secteurs culturels marchands & $5 \%$ & $2 \%$ & $1 \%$ & $0,5 \%$ & $10 \%$ \\
\hline
\end{tabular}




\section{Entre 2008 et 2011, une activité plutôt dynamique dans le spectacle vivant}

Les déclarations de TVA des entreprises permettent de calculer des indices d'évolution des chiffres d'affaires: en utilisant cette source, on constate que le chiffre d'affaires de l'ensemble des secteurs marchands des "arts, spectacles et activités récréatives » progresse de $11 \%$ entre 2008 et 2011 et les trois secteurs du spectacle vivant ont aussi été plutôt dynamiques au cours de la période (graphique 2). Le chiffre d'affaires des salles de spectacles, avec une croissance de + $10 \%$ entre 2008 et 2011, contribue fortement à l'évolution des arts, spectacles et activités récréatives. De plus, le secteur des salles de spectacles ne semble pas avoir subi la crise de 2009 qui a affecté l'ensemble des services marchands : à l'inverse des arts ou du soutien au spectacle vivant, le secteur des salles de spectacles affiche une hausse de $6 \%$ d'évolution de son chiffre d'affaires entre 2008 et 2009.

Après 2009, les activités de soutien sont celles qui ont le plus rapidement progressé, avec environ $+4 \%$ annuel entre 2009 et 2010 et entre 2010 et $2011,+9 \%$ sur la période 2008 2011.

Enfin, l'évolution du chiffre d'affaires des activités des arts du spectacle vivant est restée stable durant la période $(+0,5 \%)$, à l'instar de l'ensemble des activités de services aux ménages.

\section{Graphique 2 - Indice d'évolution du chiffre d'affaires entre 2008 et 2011}

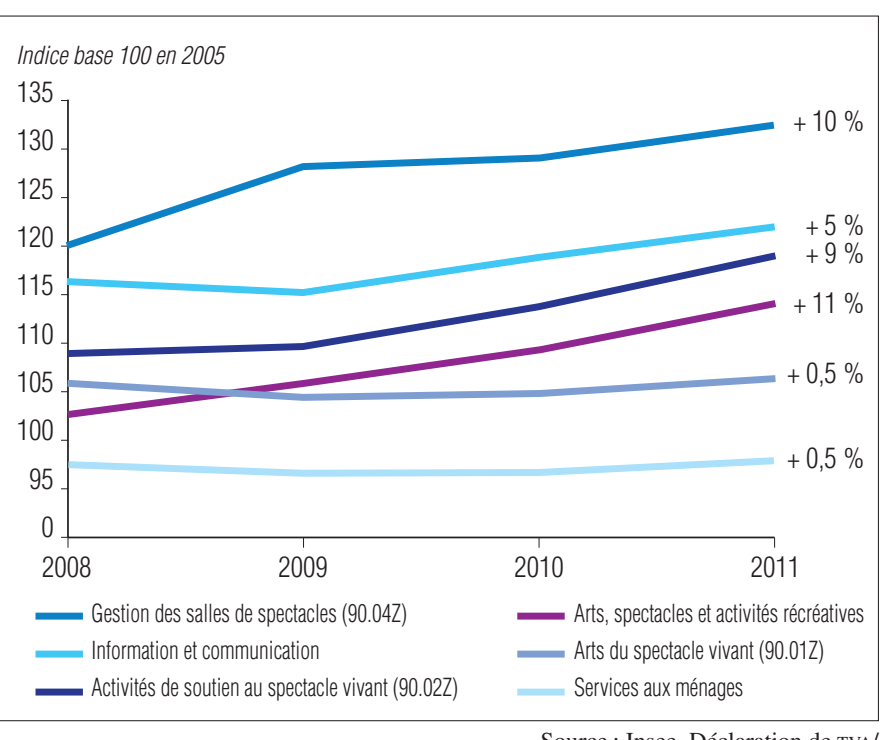

Ministère de la Culturecto et de la Communication, DEPS, 2013 publicitaires (56\%), cette proportion fait partie des charges les plus lourdes. Elles le restent aussi pour les activités de soutien $(41 \%)$ et les salles de spectacles (38\%), dans la moyenne des charges externes de l'ensemble des secteurs culturels marchands (44\%). De la même façon, les achats de matières premières, d'autres approvisionnements et marchandises sont relativement plus élevés dans les activités du spectacle vivant que dans les autres secteurs culturels : la moyenne de ces achats est de $11 \%$ alors que les achats de matières premières et autres approvisionnements représentent $26 \%$ des charges des salles de spectacles en 2010 (hors impôts, taxes et charges de personnel), $12 \%$ pour les activités de soutien et $7 \%$ pour les activités des arts du spectacle vivant. Quant aux charges de location, elles sont comparables pour les trois secteurs d'activités, d'environ $6 \%$, et sont supérieures à la moyenne des charges de location des secteurs culturels marchands $(3 \%)$.

Les autres postes de charges d'exploitation qui comprennent les redevances sur les brevets ou licences et les droits d'auteur et de reproduction ${ }^{6}$ sont de l'ordre de $2 \%$ à $3 \%$ en 2010 pour chacun des trois secteurs d'activités. Ces dernières ainsi que les charges exceptionnelles et les charges financières sont cette fois inférieures à la moyenne de celles constatées en 2010 pour l'ensemble des secteurs culturels marchands. Cependant et par comparaison, si dans la production de films pour le cinéma, la part des redevances pour droits d'auteur est de l'ordre de $11 \%$, dans le design et la photographie les taux sont du même niveau que dans le spectacle vivant, d'environ $2 \%$.

\section{Tableau 4-Part des principaux postes de charges du spectacle vivant en 2010}

(hors impôts, taxes et charges de personnel)

\begin{tabular}{|c|c|c|c|}
\hline & $\begin{array}{c}\text { Arts du } \\
\text { spectacle vivant }\end{array}$ & $\begin{array}{l}\text { Activités de soutien } \\
\text { au spectacle vivant }\end{array}$ & $\begin{array}{c}\text { Gestion de salles } \\
\text { de spectacles }\end{array}$ \\
\hline Achats de matières premières et autres approvisionnements & 7 & 12 & 26 \\
\hline Autres achats et charges externes & 51 & 41 & 38 \\
\hline Locations, charges locatives et de copropriété & 6 & 6 & 5 \\
\hline Crédit-bail & 0 & 1 & 0 \\
\hline Coût du personnel extérieur à l'entreprise & 1 & 1 & 1 \\
\hline Autres charges d'exploitation & 2 & 3 & 3 \\
\hline dont redevances pour concessions de brevets, de licences, droits d'autel & eur & 2 & 2 \\
\hline Charges financières & 0 & 1 & 0 \\
\hline Charges exceptionnelles & 1 & 1 & 1 \\
\hline
\end{tabular}




\section{Caractéristiques STRUCTURelles DU SPECTACLE VIVANT}

\section{Une faible valeur ajoutée par entreprise, mais une productivité dans la moyenne des secteurs marchands}

Les unités des secteurs du spectacle vivant sont en moyenne de petite taille - un ETP annuel par entreprise - et présentent des chiffres d'affaires et valeurs ajoutées moyens relativement modestes par rapport aux autres secteurs culturels. Ainsi, une entreprise marchande du spectacle vivant réalise en 2010 une valeur ajoutée moyenne de 80000 euros, contre 180000 euros dans le secteur culturel marchand et 310000 euros dans l'ensemble des services marchands (tableau 5). Les résultats du spectacle vivant sont toutefois supérieurs à ceux de certaines activités culturelles comme le design qui affiche des caractéristiques similaires en termes de population (environ 20000 unités en 2010 qui dégagent une valeur ajoutée de 724 millions d'euros) mais présente des ratios moyens inférieurs de moitié à ceux du spectacle vivant (40 000 euros de valeur ajoutée par structure).

En 2010, les entreprises des arts du spectacle vivant dégagent la valeur ajoutée moyenne par unité la plus faible de l'ensemble du spectacle vivant marchand: elle est de 50000 euros quand celle des activités de soutien atteint 120000 euros (tableau 4). La valeur ajoutée moyenne de la gestion des salles est trois fois supérieure à celle des activités de soutien : en atteignant 360000 euros en 2010, elle est supérieure à la valeur ajoutée moyenne des unités de tous les secteurs marchands de l'économie française (310 000 euros).

En revanche, la productivité apparente moyenne par salarié du spectacle vivant est de 70000 euros, soit un résultat comparable à l'ensemble des secteurs marchands. Elle est la plus faible dans la gestion des salles (50 000 euros par salarié) alors que celles des arts du spectacle vivant et des activités de soutien sont assez proches (respectivement 70000 euros et 80000 euros).

Ces valeurs moyennes dissimulent une disparité entre structures : parmi les trois secteurs marchands du spectacle vivant, celui des salles de spectacles est le plus homogène, sa dispersion du chiffre d'affaires et de la valeur ajoutée est la plus faible (le coefficient de variation ${ }^{7}$ de la valeur ajoutée moyenne y est de 3,5\% quand celui des arts du spectacle est de $6,9 \%$ et celui des activités de soutien de $7 \%$ ).

En effet, derrière des caractéristiques moyennes modestes comparées aux autres secteurs marchands, le spectacle vivant marchand cache une grande variété : en termes de statut juridique tel que défini par la nomenclature des catégories juridiques ${ }^{8}$, un tissu largement fourni de petites structures individuelles dans les arts du spectacle côtoient une majorité de sociétés commerciales de type SARL (société anonyme à responsabilité limitée) dans les salles de spectacles du champ marchand.

\section{Des entreprises plutôt jeunes}

Parmi les entreprises marchandes du spectacle vivant en activité en 2010, environ la moitié ont été créées entre les années 1980 et 2000 (graphique 3).

La création des entreprises des arts du spectacle et des activités de soutien s'est en particulier accélérée à partir de 1980 : 826 entreprises marchandes des arts du spectacle vivant actives en 2010 existaient déjà en 1980 ; leur nombre est passé à 6635 en 2000.

Les entreprises d'activités de soutien au spectacle vivant comptaient 123 entreprises en 1980 contre 1920 en fin d'année 2000.

En revanche, l'évolution annuelle des entreprises marchandes de gestion de salles de spectacles entre 1980 et 2000 a été plus stable, dans la mesure où le fort mouvement de créations date des années 1950 et 1960.

Tableau 5 - Indicateurs structurels moyens des secteurs du spectacle vivant en 2010

\begin{tabular}{|c|c|c|c|c|}
\hline $\begin{array}{l}\text { Secteur } \\
\text { d'activité NAF } \\
2008\end{array}$ & Libellé de l'activité & $\begin{array}{l}\text { Effectif ETP moyen } \\
\text { (ETP/nb unités) }\end{array}$ & $\begin{array}{l}\text { VA moyenne } \\
\text { (VA/nb unités) } \\
\text { (en euros) }\end{array}$ & $\begin{array}{c}\text { Productivité moyenne } \\
\text { par salarié } \\
\text { (VA/ETP) } \\
\text { (en euros) }\end{array}$ \\
\hline $90.01 Z$ & Arts du spectacle vivant & 0,73 & 50000 & 70000 \\
\hline $90.02 Z$ & Activités de soutien au spectacle vivant & 1,44 & 120000 & 80000 \\
\hline \multirow[t]{4}{*}{$90.04 Z$} & Gestion de salles de spectacles & 7,27 & 360000 & 50000 \\
\hline & Spectacle vivant marchand & 1,16 & 80000 & 70000 \\
\hline & Secteurs culturels marchands & 1,79 & 180000 & 100000 \\
\hline & Secteurs marchands & 3,69 & 310000 & 80000 \\
\hline
\end{tabular}

7. Le coefficient de variation (CV), rapport entre l'écart-type et la moyenne, sert à mesurer la dispersion autour de la moyenne. Ainsi, le CV de 3,5\% pour la valeur ajoutée moyenne des salles de spectacles signifie que pour $70 \%$ des salles, la valeur ajoutée dégagée est égale à + ou - 3,5\% de 360 000 euros. 8. La nomenclature des catégories juridiques retenue dans SIRENE®, répertoire officiel d'immatriculation des entreprises et des établissements, a été élaborée sous l'égide du comité interministériel SIRENE®. C'est une nomenclature à vocation interadministrative, utilisée dans la gestion du Registre du commerce et des sociétés. 


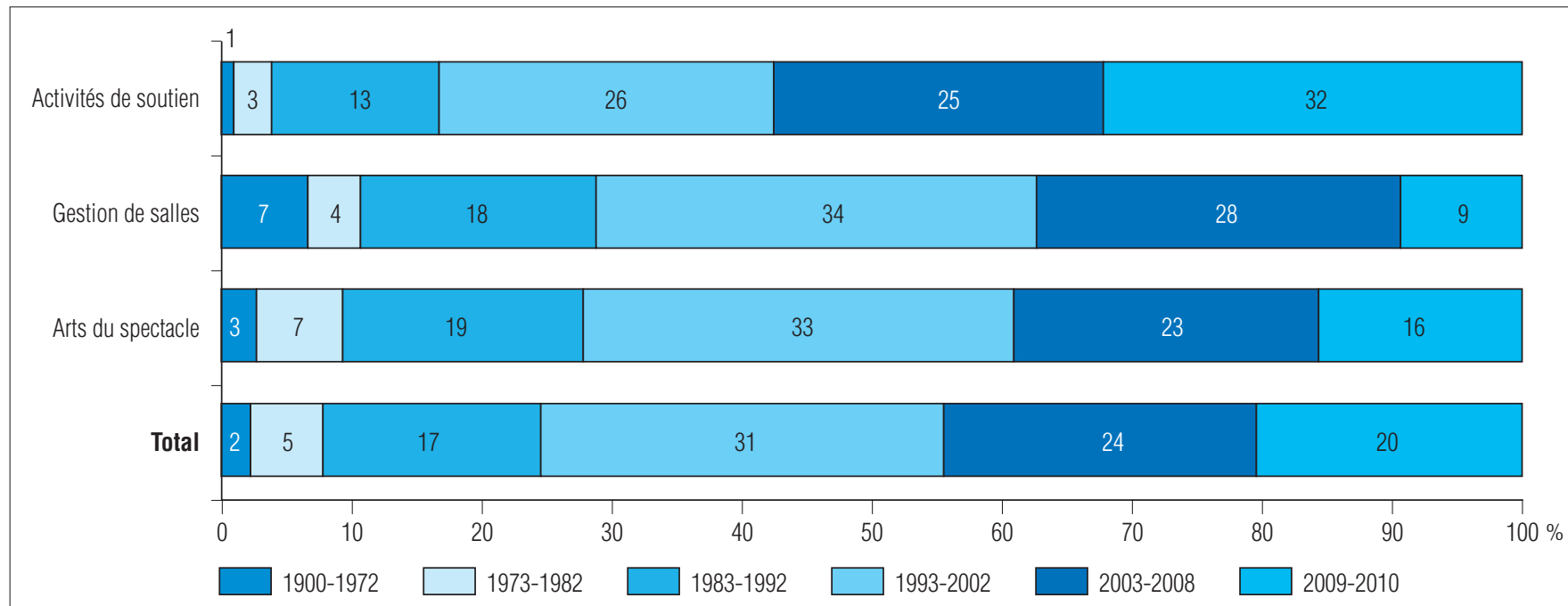

Champ : France, ensemble des unités légales des secteurs marchands, y compris auto-entrepreneurs.

Source : Insee, Ésane 2010/Ministère de la Culture et de la Communication, DEPS, 2013

Les entreprises marchandes du spectacle vivant sont ainsi relativement jeunes puisque 2 entreprises sur 5 ont moins de 10 ans en 2010 : $44 \%$ ont été créées depuis 2003. Les entreprises des activités de soutien comptent parmi les plus jeunes : $32 \%$ d'entre elles sont nées entre 2009 et 2010. Les entreprises de cette génération dans les arts du spectacle ne sont que $16 \%$. Entre 2009 et 2010, on enregistre moins de créations dans les salles de spectacles $(9 \%$ seulement).

Les entreprises marchandes du spectacle vivant sont majoritairement des sociétés commerciales ou des entreprises exerçant leur activité sous forme d'entreprise individuelle ainsi que quelques associations employeuses et marchandes ${ }^{9}$ et groupements d'intérêt économique.

Les sociétés commerciales - bien que minoritaires en nombre d'unités (31\%) en 2010 - contribuent à l'essentiel $(87 \%)$ de la valeur ajoutée produite dans le spectacle vivant marchand. Pour chacun des trois secteurs d'activités, la contribution à la valeur ajoutée des sociétés commerciales est primordiale : environ $75 \%$ dans les activités de soutien et plus de $80 \%$ pour les arts du spectacle et la gestion des salles marchandes.

En nombre d'unités, la part des sociétés commerciales est très variable : $25 \%$ dans les arts du spectacle, $43 \%$ dans les activités de soutien et jusqu'à $83 \%$ dans les salles de spectacles. Parmi l'ensemble des sociétés commerciales de spectacle vivant, les trois quarts sont des sociétés à responsabilité limitée (SARL) ; $15 \%$ ont un statut de SARL unipersonnelles et $6 \%$ de sociétés par actions simplifiées (SAS).

À l'inverse, les entreprises individuelles du spectacle vivant marchand sont les plus nombreuses en termes d'unités (67\% du total du secteur) pour une très faible contribution à la valeur ajoutée totale $(4 \%)$. En nombre, elles repré- sentent $74 \%$ du total des unités des arts du spectacle, contre $56 \%$ des unités de soutien au spectacle vivant et à peine $15 \%$ des salles de spectacles. Ce statut d'entreprise individuelle est celui par exemple d'artistes auteurs, d'animateurs artistiques, de producteur de spectacles. Les entreprises individuelles contribuent très faiblement à la valeur ajoutée des arts du spectacle et de la gestion des salles (moins de $3 \%$ en 2010); en revanche, la valeur ajoutée dégagée par les entreprises individuelles de soutien au spectacle vivant correspond à environ $20 \%$ en 2010.

D'autres entreprises considérées comme marchandes exercent leur activité sous un autre statut (association marchande ou groupement d'intérêt économique par exemple) : elles sont peu nombreuses, $2 \%$ du total des unités, et contribuent à $8 \%$ de la valeur ajoutée des arts du spectacle et à $15 \%$ de celle de la gestion des salles en 2010.

\section{Plus de $60 \%$ de la valeur ajoutée du spectacle vivant marchand est concentrée en Île-de-France}

La répartition des unités de spectacle vivant dessine une carte nettement découpée en quatre zones géographiques de la France métropolitaine : premièrement l'Île-deFrance, qui se détache largement en rassemblant $36 \%$ des unités de spectacle vivant ${ }^{10}$; ensuite le Sud-Est avec RhôneAlpes et Provence-Alpes-Côte d'Azur, régions dans lesquelles environ une entreprise de spectacle vivant sur dix est domiciliée ; troisièmement le Nord-Ouest et le Sud-Ouest, où la Bretagne, les Pays de la Loire, l'Aquitaine, MidiPyrénées et le Languedoc-Roussillon accueillent chacune $4 \%$ des unités françaises de spectacle vivant; enfin toutes

9. La très grande majorité des associations sont « non marchandes » et ne font donc pas partie du champ de cette étude. Toutefois, quelques entreprises sous statut juridique associatif sont dans le champ de l'enquête, car elles sont employeuses et leurs recettes couvrent plus de $50 \%$ de leur production. 10. L'entreprise est localisée à partir de l'adresse de son siège et dans les secteurs du spectacle vivant, la quasi-totalité des entreprises sont mono-établissements. 
Carte 1 - Localisation géographique des entreprises marchandes de spectacle vivant en 2010

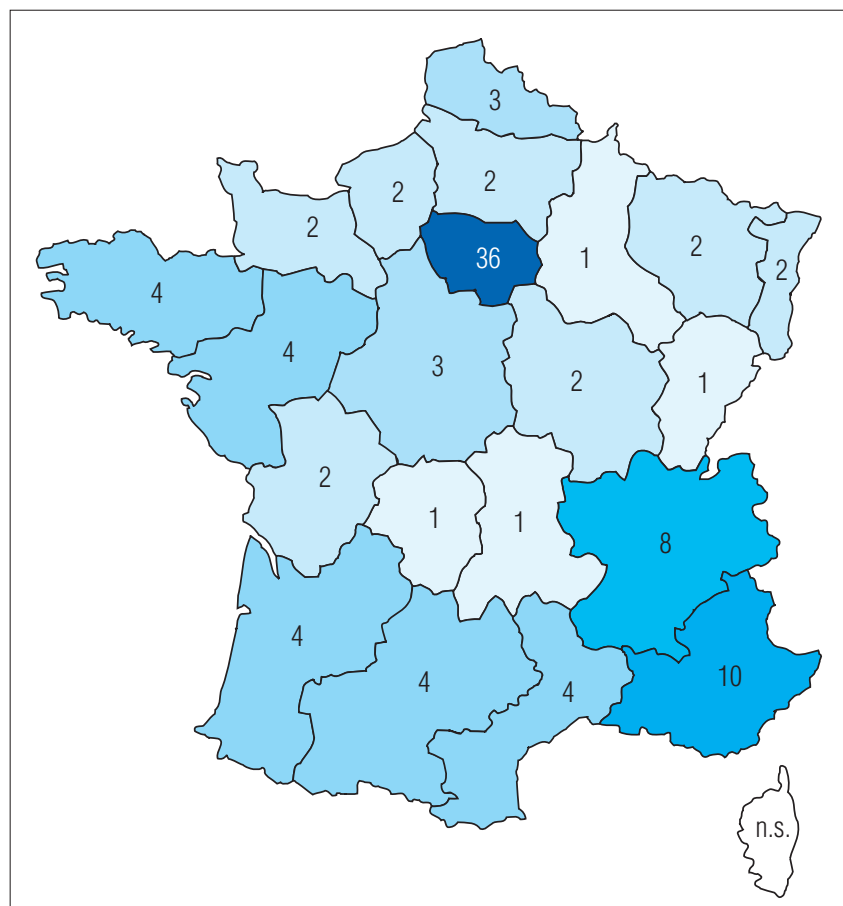

Champ: France, ensemble des unités légales des secteurs marchands, y compris auto-entrepreneurs. les autres régions abritent chacune moins de $4 \%$ des unités de spectacle vivant (carte 1).

L'Île-de-France concentre la population mais aussi la valeur ajoutée dégagée par les entreprises de spectacle vivant en 2010 : un peu plus d'un tiers des structures marchandes sont franciliennes et elles représentent plus de $60 \%$ de la valeur ajoutée de l'ensemble (tableau 6). Les deux régions Rhône-Alpes et Provence-Alpes-Côte d'Azur confirment leur importance avec des valeurs ajoutées correspondant à respectivement $6 \%$ et $7 \%$ de l'ensemble du marché.

La forte densité régionale d'unités de spectacle vivant et leur contribution économique correspond au dynamisme démographique de ces trois régions : en effet, l'attractivité de l'Île-de-France, de Rhône-Alpes et de Provence-AlpesCôte d'Azur s'exprime aussi en termes de répartition de la population vivant sur le territoire et de richesse produite.

Source : Insee, Ésane 2010/Ministère de la Culture et de la Communication, DEPS, 2013

Tableau 6-Répartition régionale : démographie et spectacle vivant en nombre d'unités et valeur ajoutée en 2010

\begin{tabular}{|c|c|c|c|c|}
\hline Région & $\begin{array}{c}\% \text { de la } \\
\text { opulation française }\end{array}$ & $\begin{array}{l}\text { Produit intérieur brut } \\
\text { régional par habitant } \\
\text { (en euros) }\end{array}$ & $\begin{array}{c}\% \text { des unités } \\
\text { de spectacle vivant }\end{array}$ & $\begin{array}{l}\% \text { de la valeur ajoutée } \\
\text { du spectacle vivant }\end{array}$ \\
\hline Alsace & 3 & 28302 & 2 & 2 \\
\hline Aquitaine & 5 & 26268 & 4 & 2 \\
\hline Auvergne & 2 & 24281 & 1 & 1 \\
\hline Basse-Normandie & 2 & 23581 & 2 & 1 \\
\hline Bourgogne & 3 & 25826 & 2 & 1 \\
\hline Bretagne & 5 & 25278 & 4 & 1 \\
\hline Centre & 4 & 25423 & 3 & 2 \\
\hline Champagne-Ardenne & 2 & 26804 & 1 & 0 \\
\hline Corse & 1 & 23870 & 0 & 0 \\
\hline Franche-Comté & 2 & 24186 & 1 & 1 \\
\hline Haute-Normandie & 3 & 26281 & 2 & 1 \\
\hline Île-de-France & 18 & 49268 & 36 & 63 \\
\hline Languedoc-Roussillon & 4 & 23653 & 4 & 2 \\
\hline Limousin & 1 & 23142 & 1 & 0 \\
\hline Lorraine & 4 & 23665 & 2 & 1 \\
\hline Midi-Pyrénées & 5 & 26576 & 4 & 2 \\
\hline Nord-Pas-de-Calais & 6 & 24507 & 3 & 2 \\
\hline Pays de la Loire & 6 & 26969 & 4 & 3 \\
\hline Picardie & 3 & 23198 & 2 & 1 \\
\hline Poitou-Charentes & 3 & 23870 & 2 & 1 \\
\hline Provence-Alpes-Côte d'Azur & 8 & 27782 & 10 & 7 \\
\hline Rhône-Alpes & 10 & 30516 & 8 & 6 \\
\hline Ensemble DOM & 3 & 18267 & 3 & 2 \\
\hline Total France & $100 \%$ & 30117 & $100 \%$ & $100 \%$ \\
\hline
\end{tabular}




\section{RÉPARTITION DU CHIFFRE D'AFFAIRES ET DES SOURCES DE FINANCEMENT}

\section{Une forte concentration sur le cour de métier}

Les chiffres d'affaires des activités du spectacle vivant reposent avant tout sur leur cœur de métier : en 2010, les trois secteurs du spectacle vivant réalisent environ $90 \%$ de leurs chiffres d'affaires dans les branches d'activités ${ }^{11}$ en lien direct avec leurs métiers centraux : de création, de production et de diffusion de spectacles, ou de soutien au spectacle vivant, en fonction de leur activité principale.

Ces résultats sont comparables à ceux d'autres secteurs dans lesquels la branche d'activité principale pèse le plus : les ventes d'éditions de journaux (93\%), les éditions de jeux électroniques $(91 \%)$ ou l'architecture $(91 \%)$.

D'autres secteurs culturels ont une concentration de leurs activités moins forte : l'édition de livres ( $76 \%$ ), la production de films pour le cinéma (78\%), le design (85\%).

Dans le spectacle vivant, le secteur des activités de soutien est le plus hétérogène : il englobe des activités de production sans responsabilité artistique mais aussi des activités généralistes (administratives, transversales) ou techniques (sonorisation, éclairage, etc.) : comme ses contours d'activités sont divers, le poids de son chiffre d'affaires (94\%) est le plus concentré dans ces mêmes activités générales dites de soutien (tableau 7).

À l'inverse, le secteur des arts du spectacle vivant, c'està-dire les créateurs et producteurs avec responsabilité artistique, est celui qui diversifie le plus ses activités vers d'autres branches d'activités, en particulier dans des activités culturelles de création artistique qui ne relèvent pas du spectacle vivant (tableau 8) : $72 \%$ du CA du secteur est réalisé dans leur branche d'activité des arts du spectacle vivant, mais aussi $11 \%$ dans la création artistique et $4 \%$ dans la création plastique.

La création artistique inclut les écrivains et journalistes mais elle comprend aussi les activités des compositeurs de musique qui se rapportent bien à des activités de création de spectacles. La création plastique s'applique aux peintres, dessinateurs, sculpteurs, graveurs, etc.

La vente de livres ou de prestations lors de salons représente à peine plus de $1 \%$ du chiffre d'affaires du secteur des arts du spectacle vivant en 2010 ; quant à l'enseignement artistique et de loisirs ou les autres activités récréatives ou de loisirs (prestations dans des parcs de loisirs, des discothèques, organisation d'événements divers), ils participent très faiblement au chiffre d'affaires du secteur (moins de $1 \%)$.

Enfin, les entreprises marchandes gérant des salles de spectacles ont réalisé, en 2010, $86 \%$ de leur chiffre d'affaires dans la gestion et l'exploitation de lieux de spectacles, $6 \%$ dans la création, production, organisation de spectacles et environ $2 \%$ dans la vente de boissons (tableau 9).
D'autres secteurs d'activités marchandes investissent aussi dans le spectacle vivant: il s'agit principalement de services d'organisation de salons professionnels et congrès, de débits de boissons et de commerces mais leur participation au chiffre d'affaires total des branches du spectacle vivant reste très faible, inférieure à $1 \%$ en 2010.

En outre, l'analyse détaillée des activités des entreprises des trois secteurs du spectacle vivant permet d'observer les spécificités de toute la chaîne, de la création à la diffusion : l'importance de la création s'affirme par le poids représenté par la production et l'organisation des spectacles euxmêmes (soit plus de la moitié des ventes de l'ensemble en

\section{Tableau 7 - Secteur du soutien au spectacle vivant (90.02Z) : répartition du chiffre d'affaires 2010 par branche d'activités les plus contributrices}

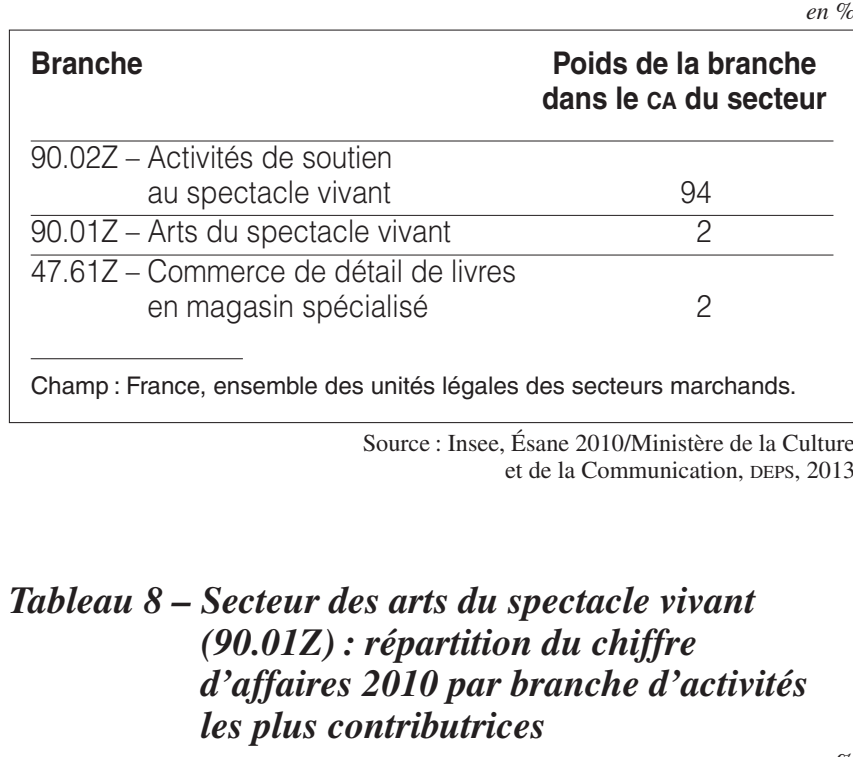

\section{en $\%$}

\begin{tabular}{|c|c|}
\hline $\begin{array}{l}\text { Poids d } \\
\text { dans le }\end{array}$ & $\begin{array}{l}\text { la branche } \\
\text { du secteur }\end{array}$ \\
\hline 90.01Z - Arts du spectacle vivant & 72 \\
\hline 90.03B - Autre création artistique & 11 \\
\hline$\overline{90.02 Z ~-~ A c t i v i t e ́ s ~ d e ~ s o u t i e n ~ a u ~ s p e c t a c l e ~ v i v a n t ~}$ & 4 \\
\hline $\begin{array}{c}\text { 90.03A - Création artistique relevant } \\
\text { des arts plastiques }\end{array}$ & 4 \\
\hline$\overline{90.04 Z ~-~ G e s t i o n ~ d e ~ s a l l e s ~ d e ~ s p e c t a c l e s ~}$ & 3 \\
\hline 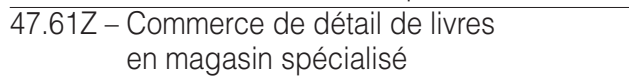 & \multirow{5}{*}{$<<5$} \\
\hline $\begin{array}{c}82.30 Z \text { - Services d'organisation de salons } \\
\text { professionnels et congrès }\end{array}$ & \\
\hline $\begin{array}{l}\text { 68.20B - Location de terrains } \\
\text { et d'autres biens immobiliers }\end{array}$ & \\
\hline 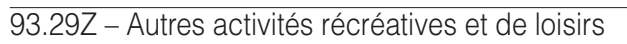 & \\
\hline $\begin{array}{r}85.52 Z \text { - Enseignement culturel } \\
\text { (artistique et de loisirs) }\end{array}$ & \\
\hline
\end{tabular}

Champ : France, ensemble des unités légales des secteurs marchands.

Source : Insee, Ésane 2010/Ministère de la Culture et de la Communication, DEPS, 2013

11. Les résultats par branche d'activités décrivent les résultats de l'activité elle-même, que celle-ci soit réalisée à titre principal ou à titre secondaire par une entreprise. Ils permettent ainsi de ventiler les chiffres d'affaires de chacun des trois secteurs d'activités du spectacle vivant et aussi de regarder si d'autres secteurs réalisent des activités de spectacles. 
Tableau 9 - Secteur de la gestion de salles de spectacles $(90.04 Z)$ : répartition du chiffre d'affaires par branche d'activités les plus contributrices en 2010

\begin{tabular}{|lc|}
\hline Branche & $\begin{array}{c}\text { en } \% \\
\text { Poids de la branche } \\
\text { dans le cA du secteur }\end{array}$ \\
\hline $90.04 Z$ - Gestion de salles de spectacles & 86 \\
\hline$\overline{90.01 Z}$ - Arts du spectacle vivant & 6 \\
\hline $90.02 Z$ - Activités de soutien au spectacle vivant & 4 \\
\hline $56.30 Z$ - Débits de boissons & 2 \\
\hline $\begin{array}{l}82.99 Z \text { - Autres services de soutien } \\
\text { aux entreprises n.c.a. }\end{array}$ & 1 \\
\hline Champ : France, ensemble des unités légales des secteurs marchands.
\end{tabular}

Source : Insee, Ésane 2010/Ministère de la Culture et de la Communication, DEPS, 2013

2010), puis par les ventes de contrats (environ $35 \%$ en 2010).

De fait, la répartition du chiffre d'affaires par activité détaillée met en avant l'activité même de création et de production de spectacles avec responsabilité artistique. Ainsi, les trois secteurs réalisent environ $60 \%$ du chiffre d'affaires global grâce aux recettes de services du spectacle vivant. Le contenu de ces activités s'entend de façon large en tant qu'activité de création, production et organisation. Il peut donc s'agir d'un côté de recettes issues de la création et de la production de spectacles artistiques par des producteurs ou par des artistes indépendants (acteurs, lecteurs, chanteurs, musiciens, danseurs, conteurs, orateurs, artistes de cirque et autres artistes du spectacle); d'un autre côté, des services qui s'apparentent plus à de l'animation musicale (soirées privées, animations en milieu scolaire ou d'entreprises...), activité souvent complémentaire pour de nombreux artistes ; ou encore des recettes de services d'organisation de spectacles et enfin de celles de billetterie ${ }^{12}$.

D'autres sources de revenus sont bien identifiées en tant qu'activités de création et de production artistique : les contrats de cession représentent le deuxième poste le plus important en termes de ressources commerciales avec, en 2010, $27 \%$ du chiffre d'affaires de l'ensemble du spectacle vivant.

Les contrats de coproduction et la production de spectacles sans responsabilité artistique participent de façon équivalente au chiffre d'affaires 2010, soit environ pour $4 \%$.

Enfin, d'autres activités dégagent des recettes qui participent plus faiblement au chiffre d'affaires de l'ensemble du spectacle vivant marchand en 2010 : contrats de coréalisation, ventes de marchandises, services de scénographie, location de salles ou de matériels techniques, services de gestion, enseignement artistique et de loisirs, etc.

\section{Un fort dynamisme des entreprises de moins de 10 salariés}

Comme dans la plupart des autres secteurs culturels marchands, le tissu économique des entreprises du spectacle vivant est constitué de petites entreprises de moins de 10 salariés équivalent temps plein : $96 \%$ des entreprises marchandes du spectacle vivant emploient moins de 10 salariés en 2010, $95 \%$ dans les activités cinématographiques, vidéo et télévision, 99 \% dans les activités de design et l'ensemble des activités artistiques.

Cependant ces petites entreprises participent plus au chiffre d'affaires de l'ensemble du spectacle vivant que certaines de leurs consœurs des autres activités culturelles : $96 \%$ des moins de 10 salariés réalisent $56 \%$ du chiffre d'affaires total. Dans les activités cinématographiques, vidéo et télévision, l'ensemble de ces entreprises de moins de 10 salariés réalise environ $30 \%$ du chiffre d'affaires de leur secteur. Dans le design, $95 \%$ des entreprises de moins de 10 salariés réalisent $68 \%$ du chiffre d'affaires du secteur.

Parallèlement, les entreprises de plus de 20 salariés du spectacle vivant ne sont que $2 \%$ en nombre mais réalisent environ $34 \%$ du chiffre d'affaires. Cette configuration est assez proche de celle du design où les entreprises de plus de 20 salariés réalisent près de $30 \%$ du chiffre d'affaires en 2010.

Des différences s'observent entre les trois secteurs du spectacle vivant (tableau 10).

Les arts du spectacle regroupent le plus d'unités sans salarié ${ }^{13}$, soit $82 \%$ du nombre total d'unités marchandes des arts du spectacle, et ces dernières participent pour $18 \%$ au chiffre d'affaires sectoriel en 2010. En y ajoutant les unités avec salariés (de 1 à 9), près de $60 \%$ du chiffre d'affaires des arts du spectacle est réalisé par des unités de moins de 10 salariés. Parallèlement, $30 \%$ du chiffre d'affaires est produit par un tout petit nombre d'unités de taille intermédiaire (de 20 à 249 salariés).

Le secteur des activités de soutien affiche une structure assez semblable en termes de population mais plus nuancée en termes de résultats commerciaux : l'immense majorité de ses structures est de petite taille ( $97 \%$ des entreprises marchandes d'activités de soutien au spectacle vivant comptent moins de 10 salariés), et elles réalisent près de la moitié du chiffre d'affaires sectoriel (48\% en 2010). Environ un tiers du chiffre d'affaires est réalisé par des entreprises de 20 à 249 salariés. Il faut noter la très faible présence des grandes entreprises mais leur participation significative au chiffre d'affaires du secteur (environ $7 \%$ en 2010).

Les entreprises marchandes de gestion de salles de spectacles dénotent une organisation différente : en effet, les entreprises de taille intermédiaire, de 20 à 249 salariés, y sont bien implantées. Ce sont principalement des entreprises de location d'espaces. Elles rassemblent $11 \%$ du

12. Pour rappel, le champ des entreprises marchandes du spectacle vivant n'inclut pas les salles nationales comme l'Opéra national de Paris, ni les lieux associatifs non marchands et non employeurs.

13. Les unités sans salarié sont celles pour lesquelles il n’y pas de document de Déclaration annuelle de données sociales (DADS) ou celles considérées sans salarié au 31 décembre (depuis 2009, les auto-entrepreneurs mono-actifs dont le chiffre d'affaires est positif durant l'année suivant leur inscription, et les auto-entrepreneurs multi-actifs - ayant, en fin d'année, à la fois une activité salariée et une activité non salariée - pour lesquels le revenu d'activité d'auto-entreprenariat est plus élevé que les revenus de l'ensemble des activités salariées de l'année). 


\begin{tabular}{|c|c|c|c|c|c|c|c|c|}
\hline & \multicolumn{2}{|c|}{ Arts du spectacle } & \multicolumn{2}{|c|}{ Activités de soutien } & \multicolumn{2}{|c|}{ Gestion de salles } & \multicolumn{2}{|c|}{ Total } \\
\hline & $\begin{array}{l}\text { Nombre } \\
\text { d'unités }\end{array}$ & $\begin{array}{c}\text { Chiffre } \\
\text { d'affaires }\end{array}$ & $\begin{array}{l}\text { Nombre } \\
\text { d'unités }\end{array}$ & $\begin{array}{c}\text { Chiffre } \\
\text { d'affaires }\end{array}$ & $\begin{array}{l}\text { Nombre } \\
\text { d'unités }\end{array}$ & $\begin{array}{c}\text { Chiffre } \\
\text { d'affaires }\end{array}$ & $\begin{array}{l}\text { Nombre } \\
\text { d'unités }\end{array}$ & $\begin{array}{c}\text { Chiffre } \\
\text { d'affaires }\end{array}$ \\
\hline De 0 à 9 & 98 & 61 & 97 & 48 & 80 & 19 & 96 & 56 \\
\hline De 10 à 19 & 1 & 8 & 1 & 12 & 8 & 12 & 2 & 10 \\
\hline de 20 à 249 & 1 & 30 & 1 & 33 & 11 & 66 & 1 & 31 \\
\hline 250 salariés ou plus & 0 & 0 & 1 & 7 & 1 & 3 & 1 & 3 \\
\hline
\end{tabular}

Sources : Insee, Ésane 2010/Ministère de la Culture et de la Communication, DEPS, 2013

nombre total d'unités marchandes de ce secteur et elles contribuent à elles seules à environ $66 \%$ du chiffre d'affaires en 2010. À l'inverse, les petites structures de moins de 10 salariés bien que toujours majoritaires en nombre (environ $80 \%$ ) ne prennent qu'environ $20 \%$ des parts du marché.

Enfin, les grandes entreprises marchandes de plus de 250 salariés sont présentes mais minoritaires en nombre et participent très faiblement aux résultats commerciaux.

La place des petites entreprises se confirme dans toute la chaîne d'activités : les plus petites entreprises, celles de moins de 10 salariés, contribuent pour $47 \%$ au montant des ventes de contrats de coproduction, pour $52 \%$ aux activités générales dites de «services du spectacle vivant », pour $55 \%$ aux contrats de coréalisation, pour $59 \%$ aux contrats de cession et jusqu'à $66 \%$ des services de production sans responsabilité artistique (graphique 4).

Seuls les droits d'enregistrement et de captation, qui, au demeurant, représentent une part faible du chiffre d'af- faires cumulé du spectacle vivant, apparaissent comme une ressource commerciale pour les structures de 20 salariés ou plus (65\% du montant).

\section{Des sources de financement complémentaires, majoritairement publiques}

En dehors des recettes commerciales liées à leurs activités, les entreprises de spectacle vivant peuvent avoir d'autres types de ressources comptabilisées: subventions publiques mais aussi soutiens financiers privés par le biais du mécénat culturel (dons, legs, etc.), parrainage (sponsoring, souvent publicitaire et qui implique une contrepartie); aides provenant d'organismes collectifs de redistribution publics ou privés : taxes et droits collectés par les sociétés de perception et de répartition des droits (SPRD), ou encore et par exemple par le Centre national des variétés, de la

\section{Graphique 4-Chiffre d'affaires des activités de spectacle vivant en 2010 : répartition par taille d'entreprise}

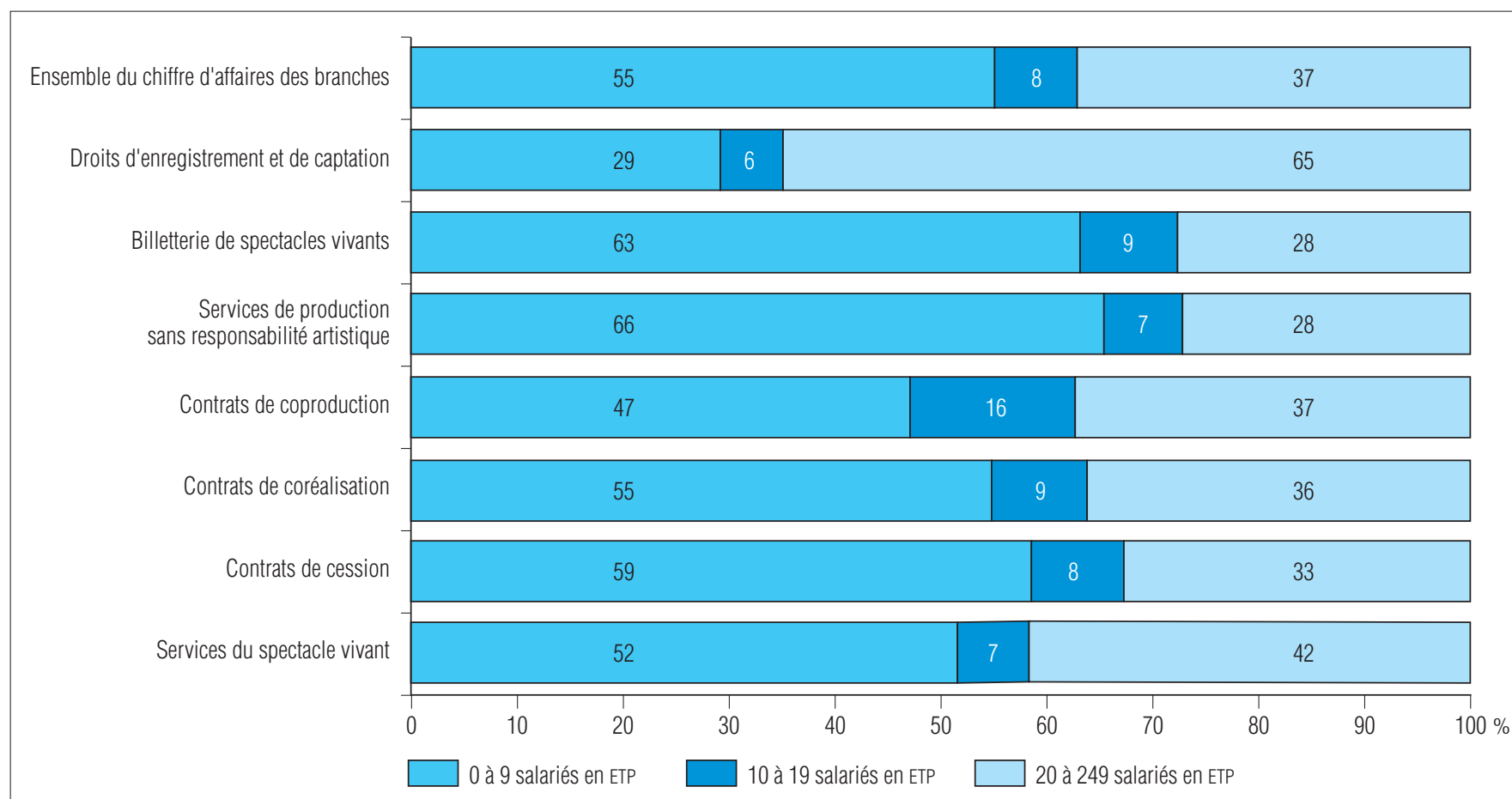

Champ : France, ensemble des unités légales des secteurs marchands, y compris auto-entrepreneurs. 
chanson et du jazz ( $\mathrm{CNV})$, le Centre national du théâtre (CNT), l'Office national de diffusion artistique (Onda), l'Association de soutien au théâtre privé (ASTP), etc.) ; enfin d'autres types de ressources (remboursement de taxes pour l'emploi de certaines catégories de publics, adhésions, etc.).

Bien que la communication et la recherche de partenaires prennent une part de plus en plus importante dans le fonctionnement économique des structures du spectacle

\section{Graphique 5 - Répartition des subventions publiques et financements privés du spectacle vivant en 2010}

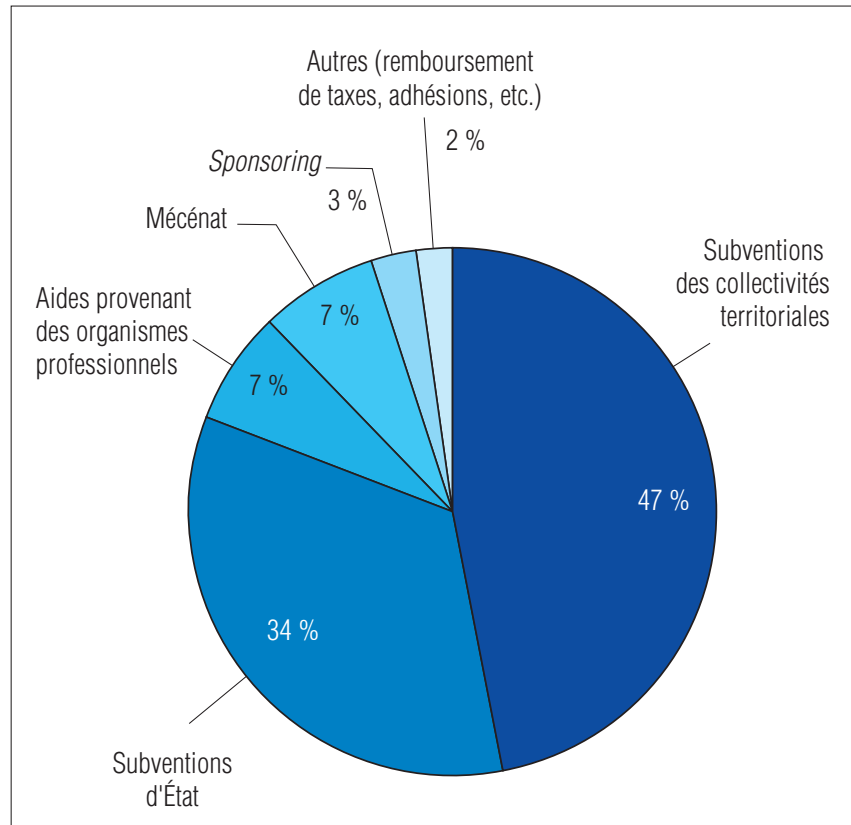

Champ : France, unités légales des secteurs marchands.

Source : Ministère de la Culture et de la Communication, DEPS, 2013 vivant, on observe que la part des subventions publiques reste l'autre source de financement prédominante.

En effet, pour les unités marchandes du spectacle vivant, la part des subventions publiques dans l'ensemble des autres financements est de $80 \%$ en 2010. Parmi ces subventions publiques, celles des collectivités territoriales sont les plus importantes : $47 \%$ des sommes déclarées en 2010 (graphique 5). Les subventions de l'État représentent un tiers du total cumulé des subventions (34\%).

Les financements privés (mécénat, sponsoring) couvrent environ $11 \%$ des ressources en 2010, hors recettes commerciales, et les organismes professionnels (organismes collectifs de redistribution publics ou privés) participent pour environ $7 \%$. Le mécénat est l'outil principal de financement privé : il correspond à $7 \%$ des sommes déclarées par l'ensemble des acteurs du spectacle vivant quand le sponsoring n'en représente que $3 \%$ en 2010.

Les trois secteurs se distinguent aussi par l'origine de leurs sources de financement : la part des subventions émanant des collectivités territoriales représente $77 \%$ des sommes déclarées des salles de spectacles, tandis que cette part ne correspond qu'à $21 \%$ des sommes perçues pour les activités de soutien et à $32 \%$ pour les arts du spectacle. En 2010, ce sont les arts du spectacle qui déclarent recevoir le plus de subventions de l'État (37\% du total de leurs sources de financement). tandis qu'elles constituent $13 \%$ des sources de financement des activités de soutien et $8 \%$ de celles des salles de spectacles.

En 2010, le mécénat est plus répandu dans les arts du spectacle et les activités de soutien: $11 \%$ des sommes déclarées, contre $2 \%$ de celles des salles de spectacles. Le sponsoring est aussi fréquent dans les activités de soutien en 2010 : il représente $11 \%$ des sommes déclarées alors qu'il ne s'élève qu'à $4 \%$ pour les acteurs des arts du spectacle et à $3 \%$ pour les salles. 


\section{Définitions (par ordre alphabétique)}

Capacité d'autofinancement (CAF) : la capacité d'autofinancement représente les ressources brutes restant à l'entreprise à l'issue de l'exercice. L'entreprise peut choisir soit de les distribuer, soit de les réserver au financement de ses investissements. Au contraire du résultat net comptable, elle ne prend pas en compte les flux se rapportant aux opérations d'appréciation ou de dépréciation du capital.

Charges : une charge correspond à un coût à supporter par l'entreprise en tant que diminution de son résultat (à l'opposé de l'écriture comptable des produits qui, eux, représentent un enrichissement, une augmentation dans le compte de résultat). La classe 6 du plan comptable général (PCG) regroupe les charges destinées à être enregistrées. Ces charges sont détaillées en différents postes, par exemple:

- les autres achats et charges externes : les achats d'études et de prestations de services, la publicité, les publications, les déplacements, les frais postaux, etc. ;

- les autres charges d'exploitation : impôts, taxes et versements assimilés, redevances pour concessions de brevets, licences, droits d'auteur et reproduction, etc. ;

- les charges financières : les charges d'intérêts d'emprunts ou bancaires, les pertes de change, etc. ;

- les charges exceptionnelles : charges qui ne sont pas caractéristiques de l'exploitation normale et ne sont pas de caractère répétitif (pénalités, amendes, créances devenues irrécouvrables, opérations d'immobilisations, etc.).

Chiffre d'affaires hors taxe (САHT): montant hors taxes des affaires réalisées par l'entreprise avec les tiers dans l'exercice de son activité professionnelle. Le chiffre d'affaires est constitué par les ventes de marchandises, la production des biens et des services (France + exportations et livraisons intracommunautaires).

Effectif salarié en équivalent temps plein : les effectifs mesurés en «équivalent temps plein » (ETP) correspondent au nombre total d'heures travaillées dans l'activité considérée divisé par la moyenne annuelle des heures travaillées dans des emplois à plein temps. Pour éviter de compter une même personne plusieurs fois, on mesure l'emploi en ETP. L'effectif salarié ETP est l'indicateur utilisé pour déterminer les tailles d'entreprises (et les contraintes légales s'y référant), ou pour établir des indicateurs (exemple : rendement de la main-d'œuvre, soit le rapport valeur ajoutée sur effectif salarié ETP).

Effectif salarié au 31 décembre : il correspond au stock de l'emploi de salariés inscrits dans l'entreprise à la date du 31 décembre.

Excédent brut d'exploitation (EBE) : autre solde intermédiaire calculé, il est la différence entre la valeur ajoutée + les subventions d'exploitation et - les impôts et taxes (sauf l'impôt sur les sociétés) et les charges de personnel supportés par l'entreprise. L'EBE représente la part de la valeur ajoutée qui revient à l'entreprise et aux apporteurs de capitaux (associés et prêteurs). II indique la ressource générée par la seule exploitation de l'entreprise indépendamment de la politique d'amortissements (dotations) et du mode de financement (charges financières).

Immobilisations corporelles : elles comprennent les biens tangibles destinés à être utilisés d'une manière durable durant le cycle d'exploitation de l'entreprise. On distingue dans cette catégorie le matériel (de transport, etc.), les équipements (de bureau, etc.), les terrains, les constructions, les installations techniques.

Immobilisations incorporelles : elles représentent les biens immatériels qui n'ont pas de substance physique, détenus par l'entreprise sur une durée supérieure à une année. Parmi ces immobilisations figurent le fonds commercial, les logiciels, le droit au bail, les brevets, les licences.

Immobilisations financières : elles comprennent les titres financiers acquis par l'entreprise pour être détenus à long terme (titres de participation) ainsi que les droits de créances à plus d'un an (prêts, dépôts et cautionnements).

Indicateur de rentabilité/Performance : les indicateurs de rentabilité correspondent à des mesures de rentabilité commerciale (EBE/CA) Ou de rentabilité économique (exemples : taux de marge, productivité du travail).

Ces indicateurs de rentabilité sont un sous-ensemble d'une notion plus large, la performance économique d'une entreprise, qui prend en considération divers résultats (exemples: les indicateurs de rentabilité, la productivité, le chiffre d'affaires, la valeur ajoutée, l'intensité d'exportation, mais aussi les résultats exprimés en termes d'objectifs atteints).

Productivité : la productivité est définie comme le rapport entre une production de biens ou services et les ressources mises en œuvre pour l'obtenir. Ces dernières sont le travail, le capital technique (installations, lieux, etc.), les capitaux engagés, les consommations intermédiaires (matières premières comme l'énergie, ainsi que sous-traitance, publicité, déplacements, etc.) mais aussi des facteurs moins faciles à appréhender bien qu'extrêmement importants, tel le savoir-faire accumulé.

Productivité apparente du travail : elle ne tient compte que du seul facteur travail comme ressource mise en œuvre. Le terme « apparente » rappelle que la productivité dépend de l'ensemble des facteurs de production et de la façon dont ils sont combinés.

Résultat courant avant impôt : il est égal au résultat d'exploitation majoré des produits financiers et minoré des charges financières + opérations en commun (bénéfice attribué) - opérations en commun (perte supportée). Il mesure la performance de l'activité économique autant que financière de l'entreprise sans intégrer les opérations exceptionnelles ou fiscales qui modifient les résultats.

Résultat net comptable (RNC) : il mesure les ressources nettes (après prise en compte de la dépréciation du capital) restant à l'entreprise à l'issue de l'exercice : bénéfice ou perte. Il correspond, au sens comptable, à la différence entre les produits et les charges de l'exercice.

Taux de marge : rapport de l'excédent brut d'exploitation sur la valeur ajoutée, il mesure la rentabilité en précisant la part de la valeur créée par l'entreprise qui sert à rémunérer les apporteurs de capitaux et à renouveler le capital investi.

Unité légale : l'unité légale est une entité juridique de droit public ou privé. Cette entité juridique peut être : 1) une personne morale, dont l'existence est reconnue par la loi indépendamment des personnes ou des institutions qui la possèdent ou qui en sont membres;2) une personne physique, qui, en tant qu'indépendant, peut exercer une activité économique.

Valeur ajoutée hors taxe - y compris autres produits et autres charges : différence entre la production globale de l'entreprise (marge commerciale + production de l'exercice) et les consommations de biens et de services en provenance des tiers + autres produits - autres charges. La valeur ajoutée mesure la capacité d'une entreprise à générer de la richesse et donc à se maintenir et se développer. Cette richesse se répartit entre plusieurs partenaires de l'entreprise : les salariés (salaires et charges sociales), l'État (par le biais des impôts et taxes), les banques (charges financières), l'entreprise elle-même (autofinancement : bénéfice net, dotation aux amortissements et aux provisions). 


\section{RÉSUMÉ}

En 2010, les entreprises marchandes qui exercent leurs activités dans les secteurs des arts du spectacle vivant, dans le soutien au spectacle vivant et dans la gestion des salles de spectacles réalisent un chiffre d'affaires de 4,4 milliards d'euros et dégagent une valeur ajoutée de près de 1,5 milliard d'euros.

L'intégration nouvelle de ces secteurs du spectacle vivant dans le dispositif des statistiques structurelles d'entreprises de l'Insee permet de les situer dans l'économie marchande française : ainsi les entreprises du spectacle vivant, hors unités majoritairement subventionnées qui sont non marchandes et associations non-employeuses, contribuent à $11 \%$ du nombre des unités des secteurs culturels marchands, à $5 \%$ de leur valeur ajoutée et à $3 \%$ de leur excédent brut d'exploitation.

La rentabilité comptable des secteurs du spectacle vivant est positive en 2010, alors que leurs charges d'exploitation sont élevées et leur taux de marge relativement inférieur à la moyenne des secteurs culturels marchands. Les secteurs du spectacle vivant marchand sont composés d'un tissu important de petites entreprises principalement installées en Îlede-France : $96 \%$ des entreprises de moins de 10 salariés réalisent $56 \%$ du chiffre d'affaires total par l'exercice de leur activité principale de création, production, diffusion et soutien au spectacle vivant.

\section{ABSTRACT}

In 2010, the market-oriented companies whose activities belong to the sectors of production of performing arts, of support activities to performing arts and of operation of arts facilities generate a 4,4 billion Euros turnover and an added value of about 1,5 billion Euros.

The recent integration of these performing arts' sectors among the Insee ${ }^{*}$ national surveys of the structural business statistics allows to reconcile them to the French market-oriented economy: the performing arts'companies - excluding companies which are mainly subsidized and considered as non market-oriented and non-profit organisations with no employees - contribute to $11 \%$ of the number of units of the market-oriented cultural sectors, to $5 \%$ of their added value and to $3 \%$ of their gross operating surplus.

Their accounting profitability is positive in 2010, while their operating expenses are significant and their margin rates relatively lower than the average of the market-oriented cultural sectors. The performing arts sectors consist of small companies mainly located in the Île-de-France territory: $96 \%$ of small companies under 10 employees realize $56 \%$ of the whole turnover with their main activity of creation, production, distribution and support for performing arts.

* Institut national de la statistique et des études économiques (Insee): National Institute of Statistics and Economic Studies.

\section{Tous les documents publiés par le DEPS sont téléchargeables sur http://www.culturecommunication.gouv.fr/Etudes-et-statistiques et sur www.cairn.info}

Pour recevoir régulièrement les publications du DEPS et pour toute demande d'information:

contact.deps@culture.gouv.fr 